$$
\text { TID/SNA - } 1734
$$

\title{
$150-f-4 a b-1$
}

I\&C SYSTEM DESIGN SUMMARY

N8600R:72-01I

ENGINEERING OPERATIONS REPORT

APRIL 1972 


\section{DISCLAIMER}

This report was prepared as an account of work sponsored by an agency of the United States Government. Neither the United States Government nor any agency Thereof, nor any of their employees, makes any warranty, express or implied, or assumes any legal liability or responsibility for the accuracy, completeness, or usefulness of any information, apparatus, product, or process disclosed, or represents that its use would not infringe privately owned rights. Reference herein to any specific commercial product, process, or service by trade name, trademark, manufacturer, or otherwise does not necessarily constitute or imply its endorsement, recommendation, or favoring by the United States Government or any agency thereof. The views and opinions of authors expressed herein do not necessarily state or reflect those of the United States Government or any agency thereof. 


\section{DISCLAIMER}

Portions of this document may be illegible in electronic image products. Images are produced from the best available original document. 


\section{I\&C SYSTEM DESIGN SUMMARY}

N8600R:72-01I

\section{ENGINEERING OPERATIONS REPORT}

W. P. Gilles

\section{MASTER}

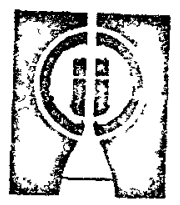

APRIL 1972

This report was prepared as an account of work sponsored by the United States Government. Neither the United States nor the United States Energy Research and Development Administration, nor any of Research and Development Administration, nor any of
their employees, nor any of their contractors, subcontractors, or their employees, makes any warranty, express or implied, or assumes any legal liability or responsibility for the accuracy, completeness or usefulness of any information, apparatus, product or process disclosed, or represents that its use would presents that infringe privately owned rights.

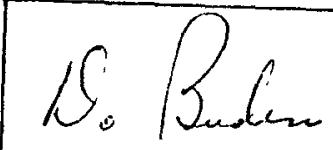

E. A. Sheridan, Manager

Controls \& Instrumentation Section Engineering Operations

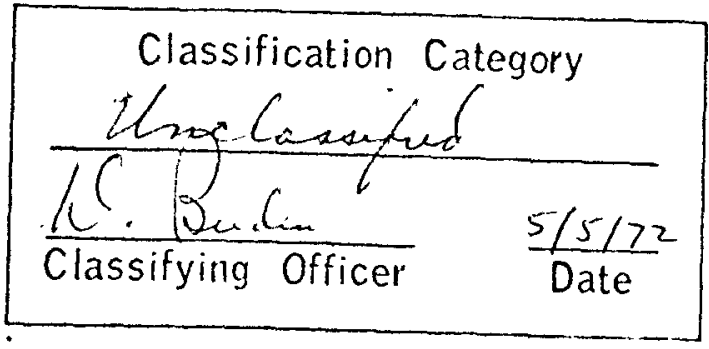


$\begin{array}{ll}1.0 \text { Introduction } & 1\end{array}$

2. Summary 1

$\begin{array}{ll}3.0 \text { Technical Discussion } & 1\end{array}$

3.1 I\&C System/Stage Interface 1

3.1.1 NERVA Functional Interfaces 2

3.1.2 Physical Interfaces 5

3.2 I\&C System Component Functional Description and $\begin{array}{ll}\text { Preliminary Interface Definition } & 7\end{array}$

3.2.1 Summary \& Conclusions 7

$\begin{array}{lll}\text { 3.2.2 Technical Discussion } & 9\end{array}$

3.3 Engine Power Requirements 36

3.4 Design Requirements 39

3.5 Electrical Interface Drawings . 39

$\begin{array}{lll}3.6 & \text { Reliability } & 39\end{array}$

$\begin{array}{ll}\text { References } & 40\end{array}$

APPENDICES

Appendix A - Data Acquisition System Design Sheet

Appendix B - Data Control Unit Design Sheet

Appendix C - Power Distribution Unit Design Sheet

Appendix D - Actuator Driver Unit Design Sheet

Appendix E - I\&C System Component Functional Block Diagrams 


\subsection{INTRODUCTION}

This report constitues the design, as of March 1972, for the NERVA Instrumentation and Control System including block diagrams of the system and its components, a set of preliminary electrical interface drawings and preliminary design sheets for several components.

\subsection{SUMMARY}

The Instrumentation and Control (I\&C) System was designed to have a simple one-wire functional interface with the nuclear stage. Simple commands such as "precondition", "start", and "shutdown" were all that would be required by the engine.

The system was designed with a self-contained battery that permitted the engine to operate without imposing electrical energy demands on the state except during the latter portion of the pulse cooldown and during coast.

Only the Data Acquisition System, the Data Control Unit, the Power Distribution Unit, and the Actuator Driver Unit were actively worked on during 1971 and 1972. Block diagrams and preliminary specifications for these components were completed and are included in this report.

System design progressed to the point that it was concluded that the system was practical from a hardware viewpoint and that there was hope of meeting the reliability requirements.

\subsection{TECHNICAL DISCUSSION}

\subsection{INSTRUMENTATION AND CONTROL SYSTEM/STAGE INTERFACE}

The following is a preliminary definition of the NERVA I\&C Systemto-Stage interfaces. These interfaces are broken into two levels of treatment; (1) functional, which relate to the general function being performed across the interfaces, and (2) physical, which cover the interconnection electrically 
of certain parts of the NERVA and the Stage. The physical interfaces result from the fact that the NERVA is assumed to be delivered in four separate pieces; (1) engine proper, (2) I\&C system electronics, (3) cooldown modules, and (4) PSoVs.

\section{3,1.1 NERVA Functiona1 Interfaces}

3.1.1.1 Power into NERVA - Power required by NERVA is discussed in Para 3.3 of this report. This profile is for 28 volts DC. In addition, if available, two references are desired: (1) 5.000 volts DC for voltage standardization, and (2) one or more AC frequency references for synchronizing purposes. A $400 \mathrm{~Hz}$ signal would be useful, as would be 1,000 or 5,000 Hz. For data synchronization purposes, another frequency is required.

\subsubsection{Commands into NERVA - These commands are assumed} to be presented to NERVA over a single wire with address and identification information. Commands are required for a number of different operations.

\subsection{Engine Operation}

3.1.1.2.1.1 Precondition engine

3.1.1.2.1.2 Start engine

3.1.1.2.1.3 Set thrust to

3.1.1.2.1.4 Shut engine down.

\subsection{Thrust Vector System}

3.1.1.2.2.1 Increase plus yaw

3.1.1.2.2.2 Increase minus yaw

3.1.1.2.2.3 Increase plus pitch

3.1.1.2.2.4 Increase minus pitch. 
3.1.1.2.3 Checkout

$\begin{array}{ll}3.1 .1 .2 .3 .1 & \begin{array}{l}\text { Perform engine } \mathrm{c} / \mathrm{o}- \\ \text { preoperation }\end{array} \\ 3.1 .1 .2 .3 .2 & \begin{array}{l}\text { Perform engine } \mathrm{c} / \mathrm{o}- \\ \text { inoperation }\end{array} \\ 3.1 .1 .2 .3 .3 & \begin{array}{l}\text { Perform engine } \mathrm{c} / \mathrm{o}- \\ \text { postoperation. }\end{array}\end{array}$

$3.1 \cdot 1.2 .4 \quad$ Status

$\begin{array}{ll}3.1 .1 .2 .4 .1 & \text { Provide all data } \\ 3.1 .1 .2 .4 .2 & \text { Provide critical data } \\ 3.1 .1 .2 .4 .3 & \text { Provide display data } \\ 3.1 .1 .2 .4 .4 & \begin{array}{l}\text { Provide state of health } \\ \text { data }\end{array} \\ 3.1 .1 .2 .4 .5 & \text { Provide time to start } \\ 3.1 .1 .2 .4 .6 & \text { Predict time of next } \\ & \text { cooldown pulse } \\ 3.1 .1 .2 .4 .7 & \begin{array}{l}\text { Predict time of cooldown } \\ \text { completion. }\end{array}\end{array}$

\title{
3.1.1.2.5 Destruct
}

3.1.1.2.5.1 Perform destruct function.

\subsubsection{Data into NERVA - This data is assumed to be}

presented to NERVA over a single wire (as in IBM data bus concept) with address and identification information. The following is a preliminary list of data required by the NERVA for proper operation.

\author{
3.1.1.3.1 Calculated I \\ 3.1.1.3.2 Calculated thrust \\ 3.1.1.3.3 Other
}




\subsubsection{Data from NERVA to Stage - This data is assumed}

to be presented to the stage over a single wire (as in the IBM data bus concept) with address and identification information. Any wide band data is considered separately.

\subsection{A11 Data - Al1 engine data for telemetry}

or other needs will be provided on demand. Some of this data is listed on the preliminary flight measurement requirement list. Other functions will be determined and will be a part of a full frame of data. All data (except wide band) will be digital and each word will occupy either a unique spot in a data frame or will have word identification included. Wide band data will be handled over separate signal lines and will be in the form of IRIG FM subcarriers.

\subsection{Critical - A limited quantity of data} words with information critical to engine operation will be provided on demand. Each word will be identified.

\subsection{Display Data - Data required for display}

will be provided on demand. Either each word will be identified or display parameters will be in a subframe of all data and each word will occupy a unique location in the subframe which also will be identified.

\subsection{State-of-Health Data - Data indicating} NERVA (engine proper and I\&C System) state-of-health will be provided on demand. This data will include redundancy remaining, impending failure in terms of trends, etc. Either a subframe of all engine data will be used with a unique position for each word, or each word, will carry its own identification.

\subsection{Time to Start - On demand, the calculated} time required to elapse before the engine may be started will be provided. 


\subsection{Time to Next Cooldown Pulse - On demand,} the calculated time until the next cooldown pulse will occur will be provided.

\subsection{Time to Cooldown Completion - On demand,} the calculated time to the completion of cooldown will be provided.

\subsubsection{Physical Interfaces}

These interfaces, for the pruposes of the I\&C System are basically the NERVA/stage harness interconnections and the I\&C electronics/stage mechanical interfaces.

3.1.2.1 NERVA-to-Stage (I\&C Electronics-to-Stage)

3.1.2.1.1 Electrical

3.1.2.1.1.1 Power Input -

TBD connectors TBD

pins each.

3.1.2.1.1.2 Command Input - two

connectors, three pins

each.

3.1.2.1.1.3 Data Input - two connectors,

three pins each.

3.1.2.1.1.4 Data Output - Two connectors, six pins each.

3.1.2.1.1.5 Structural.

3.1.2.1.2 Mechanica1

3.1.2.1.2.1 Structural - Details to be determined.

3.1.2.1.2.2 Thermal Conditioning -

To be determined. 
3.1.2.1.2.3 Preflight Purge - To be determined.

3.1.2.2 NERVA-to-NERVA Intrastage Harness

3.1.2.2.1 Propellant Shutoff Valve (PSOV)

3.1.2.2.1.1 Electrical Actuators -

TBD connectors TBD

pins each.

3.1.2.2.1.2 Instrumentation - TBD

connectors TBD pins

each.

3.1.2.2.2 Cooldown Module

3.1.2.2.2.1 Electrical Actuators -

TBD connectors TBD

pins each.

3.1.2.2.2.2 Instrumentation -

TBD connectors TBD

pins each.

3.1.2.2.3 I\&C Stage Mounted Electronics

3.1.2.2.3.1 TBD connectors TBD

pins each.

3.1.2.2.4 NERVA (Aft of Station Zero)

3.1.2.2.4.1 TBD connectors TBD

pins each. 


\subsection{I\&C SYSTEM COMPONENT FUNCTIONAL DESCRIPTION AND PRELIMINARY INTERFACE DEFINITION}

This section documents the I\&C system component interface definition and I\&C system component description. The components were defined in Specification EC-90214A. Each component is described in a functional sense with technical tradeoffs identified where more than one approach is applicable to the system concept. Preliminary design of the Power Distribution Unit, Actuator Drivers, Data Control Unit and Data Acquisition Unit components will proceed using these descriptions as a base.

\subsubsection{Summary and Conclusions}

Interface definitions and component descriptions were prepared using flow charts of data and control functions from the sensors through the computers and actuators. Component block diagrams within the flow charts were identified and are functionally described along with the interface data required to interconnect the components into a system. Following is a sumnary of each component description.

1. Engine Sensors are described by type with subgroupings according to function, control, trend or malfunction detection.

$$
\text { 2. Data Acquisition System description is divided }
$$

into two major sections, Conditioning and Sampling. Conditioning treats the problem of measured sensor voltage output modification into a signal, usable for conversion to a digital format. The Sampling section describes time division multiplexing and analog to digital conversion processes required before the Contro1 Computer can operate on any measured data.

3. The Data Control Unit description reveals a middleman function of this component. Instructions are received from the Control Computer and are decoded and passed on to the using components. Raw data is 
received from the Data Acquisition System and, after reformatting, is passed on to the Control Computer for verification and use in control system calculations.

4. The Control Computer description outlines the performance required and some limits on size of the major sections of the Control Computer; input-output processors, central processor registers, interrupts, timing, speed and memory.

\section{Stage/Vehicle/Engine Interface Unit is also}

described as a middle man; this time, between the vehicle data bus and the Control Computer.

6. Actuator Drivers are described as a component to provide the format and power to drive the engine control actuators when a signal from the control system is received.

7. The Power Distribution Unit function is described as a power load selection process when queued by the Data Control Unit.

8. The Instrumentation and Controls Weight and Volume Study contained in Reference $A$ is used as a source for description of the Harness Assembly.

9.. The Software description is divided into seven sections with a functional description of each; Executive, data acquisition, calculations, controls, outside communications, system check, and simulation.

The maturity level of the Measurements Requirements List is a constraining factor in developing detail component descriptions, in that system bandwidth is a function of the number and types of measurements and control functions required. The design of each component is dependent on the system bandwidth for the determination of techniques used and through put across the component/system interfaces. 
A major conclusion is that the software is the component that will require the greatest effort. In order to have a complete system for ground testing and subsequent flight operations, the software development cycle should be coincident with I\&C hardware development. This finding is in basic agreement with the Shuttle Program and the Apollo Program. Both of those programs are on record as recommending software design be performed in parallel with the hardware design. A minor conclusion is that a realistic look at the accuracies required for control functions be made in order to simplify the Data Acquisition System processes required to implement the I\&C System.

\subsubsection{Technical Discussion}

\subsubsection{I\&C System Component Interface Definition}

The interface definition block diagram, Figure

3.2.2.1.1, shows the components listed in Specification EC-90214A (Reference B). The heavy lines between components represent the Harness Assembly as a component interfacing with all the remainder of the components. Software is shown with a dotted line tied to the control computer to represent its residency during nonoperating periods.

Interface functions for interconnected components are defined by the data associated with each section of the Harness Assembly.

\subsubsection{I\&C System Component Descriptions \\ Specification Number EC-90214A defines nine I\&C}

system components. These are:

1. Engine Sensors

2. Data Acquisition System

3. Data Control Unıt

4. Control Computer 


\section{Vehicle Data Bus}

Commands to I\&C System Data to Stage / Vehicle

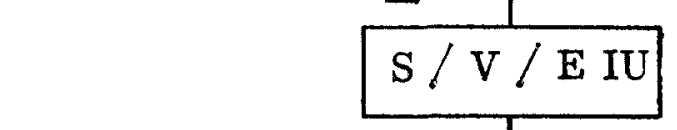

1. Raw Data to DCU-ín Serial Binary Format

2. Multiple Instructions to DAS

3. Calibrate Instructions to DAS

4. DCU Performance Data to DAS

Commands to $\mathrm{C} \overline{\mathrm{C}}$ Data to S/V/E IU

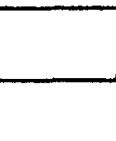

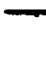

7

E S
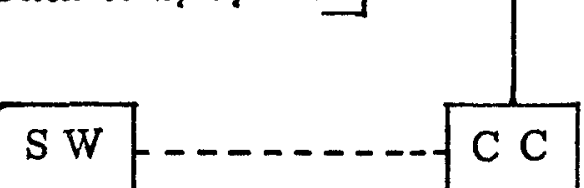

COMPONENT I D

A - ACTUATORS

AD - ACTUATOR DRIVERS

CC - CONTROL COMPUTER

DAS - DATA ACQUISITION SYSTEM

DCU - DATA CONTROL UNTT

ES - ENGINE SENSORS

PDU - POWER DISTRIBUTION UNIT

S/V/E IU - STAGE/VEHICLE/ENGINE INTERFACE UNIT

SW - SOFTWARE

1. System Timing to DCU

2. Raw Data to CC in CC Format

3. Commands to DCU

1. Power Switching Instructions to PDU

2. $\mathrm{AD}$ Instructions to PDU for 18 Drum \& 8 Valve $A D$

3. Power to DCU
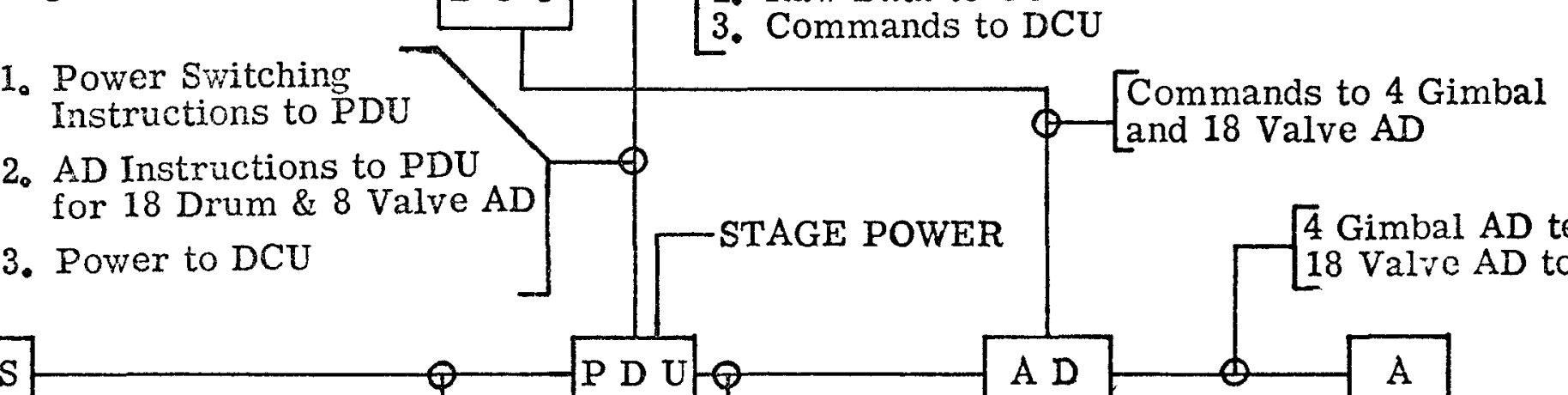

STAGE POWER

\section{Analog Data to DAS}

Bilevel Data to DAS

AD Performance

Data to DAS

I \& C SYSTEM COMPONENT INTERFACE DEFINITION BLOCK DIAGRAM

Power to DAS

2. PDU Performance Data to DAS
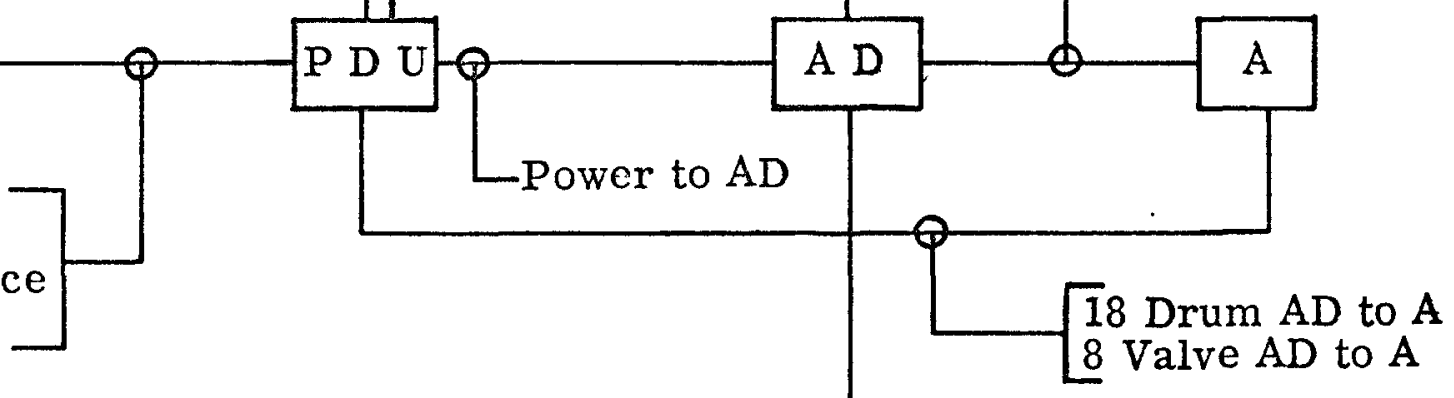

Figure 3.2 .2 .101 
5. Stage/Vehicle/Engine Interface Unit

6. Actuator Drivers

7. Power Distribution Unit

8. Harness Assembly

9. Software.

The functional characteristics of each component and the intrafaces required for the components to perform as the I\&C follows.

A. ENGINE SENSORS

1. Genera1

Engine Sensors are divided into three measurement sections:

control, trend data, and emergency malfunction detection; however, in reality all three types of measurements are used for control purposes whether it is for the immediate on-line control or as an over-ride control function. An example of this application is the event of a trend measurement not influencing engine control for long periods of time. However, if a trend data measurement indicates a modification of control is required, due to the aging or wearing out of a component, the trend data is applied and modifies (adapts) the control system to the new conditions. Emergency malfunction detection data also modifies the basic control functions if data is acquired that dictate some type of an emergency control action. The current measurements requirements list separates the engine sensors into the three categories above, and provides a justification for each measurement.

2. Engine Sensors by Major Measurement Parameter Major measurement categories are temperature, pressure, binary commands, neutronics, vibration and speed, and position. The C-101 Preliminary F1ight MRL (Reference C) details the quantities, end use functions, and performance requirements for the flight engine. 


\section{Sensor Characteristics}

The functional characteristics of the Engine Sensors will be listed in the individual sensor specifications by transducer type. To be included in this section are data on conditioning, connectors, physical specifications, environment and other data sufficient to provide a complete listing of engine sensor description.

\section{Interface}

The Engine Sensors physical interface is with engine hardware and the harness assembly. The electrical interface characteristics are defined by the signal conditioning components.

B. DATA ACQUISITION SYSTEM

1. Genera1

The data contained in this component description document supplements the information of Reference D. The data contained in that document will differ in some respects from the data contained below; in cases of differences the new data contained in this document description will take precedence. The referenced document discusses the radiation hardening problem and the location of the data acquisition system components.

\section{Conditioning}

\section{a. General}

To prepare the data generated by Engine Sensors for sampling and subequent encoding for use within the computer as control data, some conditioning is required. This section describes the conditioning required for each of the measurment types considered; temperature, pressure, neutronic, binary, vibration, and position. 


\begin{abstract}
b. Temperature
Temperature data will be gathered using two types of sensors: thermocouples and resistance temperature transducers. Conditioning of data from each of these devices will be described separately.
\end{abstract}

\title{
(1) Thermocouple Conditioning
}

The simple block diagram shown in Figure 3.2.2.2.1 includes a thermocouple, reference junction, calibration, amplifier filter, and linearization. These functions may not all be required to condition thermocouple data.

A thermocouple reference junction will be required.

Whether the reference junction is to be a temperature controlled unit, or a floating reference whose temperature is measured, is to be determined by a trade study.

Calibration of thermocouple data channels is performed by substitution of a voltage in place of the thermocouple.

Amplification of the thermocouple data will be necessary if the multiplexer (MUX) requires high level input data. If the multiplexer can use a low level data input, no amplification will be required. Filtering will be used as required to remove noise or electromagnetic interference from the thermocouple transmission line.

Thermocouple output voltage is not a linear function of temperature. Linearization can be performed within the analog signal conditioning or within the digital domain after the thermocouple data has been sampled and converted to a digital code and entered into the computer.

\section{(2) Resistance Temperature Transducer (RTT) Conditioning}

Conditioning for the resistance temperature transducer is shown in the simplified block diagram below. Included are a temperature 
sensor element, power supply, calibration medium, filter as necessary, and an amplifier. These are shown in Figure 3.2.2.2.2.

The function of the power supply is to provide a stimulus to the temperature sensor to detect changes in resistance which are the analog of the changes in temperature.

Calibration of the resistance temperature device is accomplished using a substitution resistance to simulate a change in temperature. The amplification and filter items are the same as in the thermocouple paragraph above.

\section{c. Pressure}

Pressure sensing elements generally used in the NERVA Program make use of a strain gage bridge as the conversion medium from pressure to voltage. To provide conditioning of the signal voltage provided by the bridge, items inclured in the block diagram of Figure 3.2.2.2.3 are required. These include the strain gage bridge within the transducer, a power supply, a calibration medium and an amplifier and filter. A power supply is required to provide a voltage source to the bridge such that an unbalance caused by a measurement pressure can provide an output voltage. The output voltage is usually in the millivolt range and will require amplification before multiplexing, assuming high level multiplexing. Calibration and filtering, as required, can be included within the amplifier network. The calibration can be accomplished by shunting the bridge, or voltage substitution. The filter will be applied as requircd in either a psssive mode or an active mode.

\section{d. Neutronic}

Neutronic signal conditioning requires the components shown in the simplified block diagram of Figure 3.2.2.2.4. These are neutron detectors, high voltage power supplies, amplifiers, and count rate meters. The 


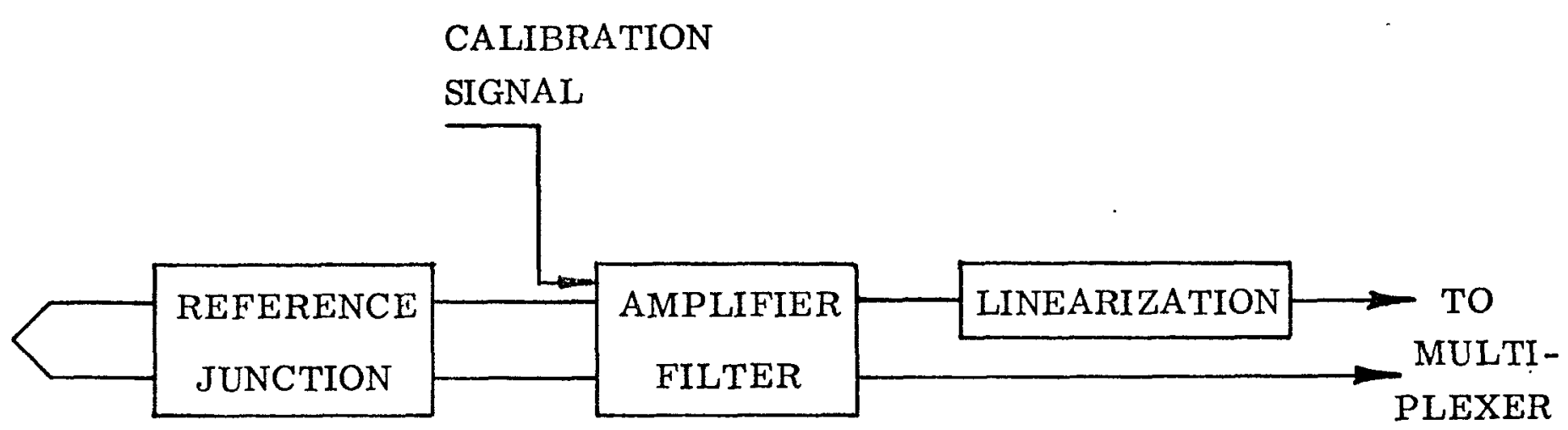

Figure 3.2.2.2.1

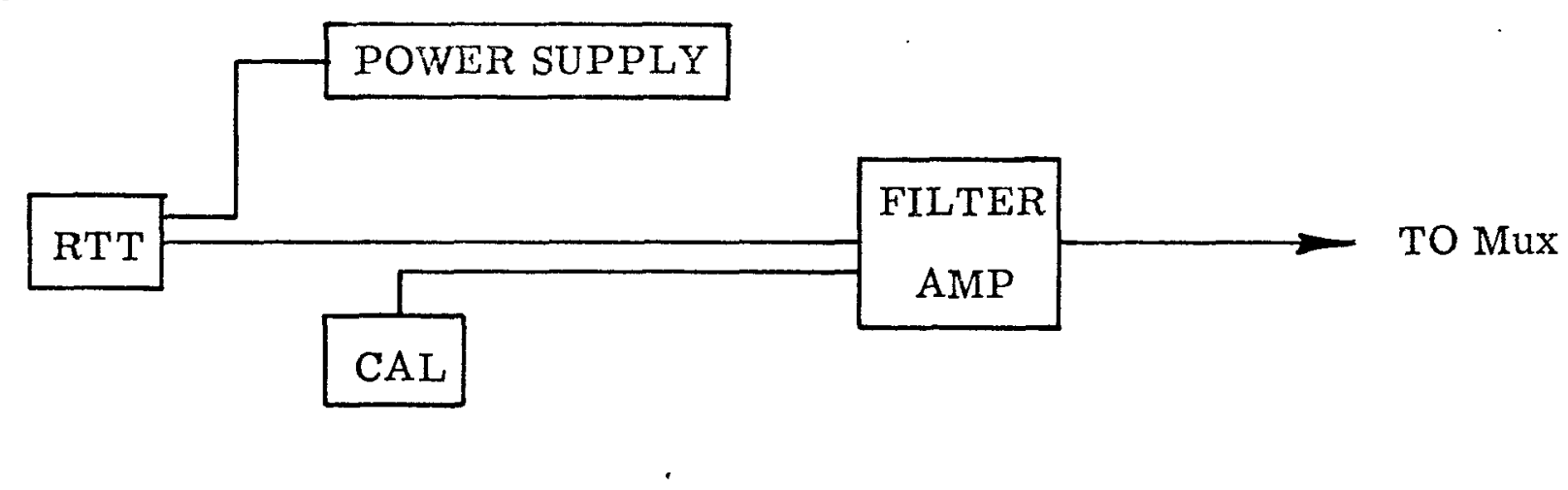

Figure 3.2.2.2.2

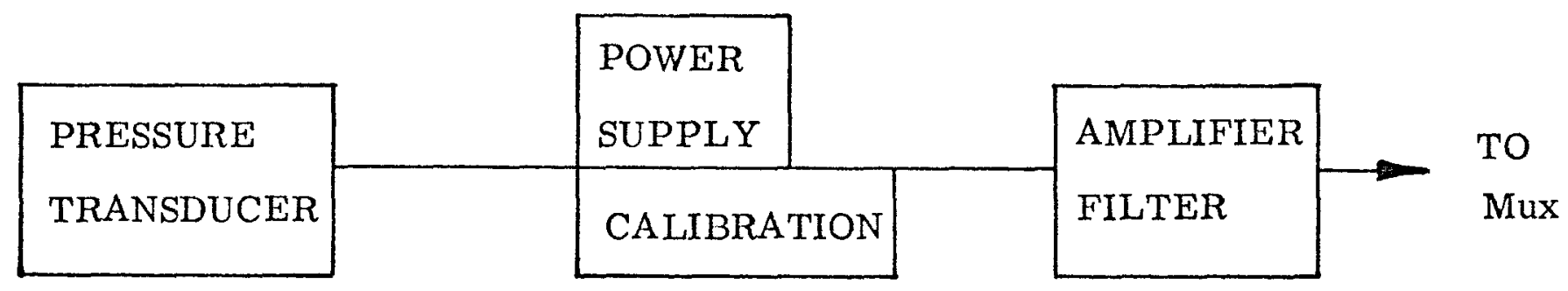

Figure 3.2 .2 .2 .3 


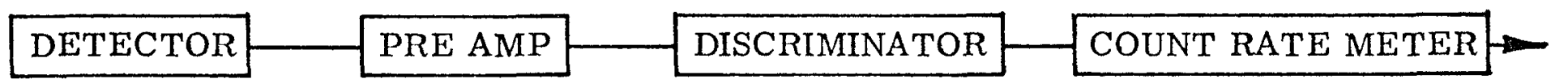

LOW POWER

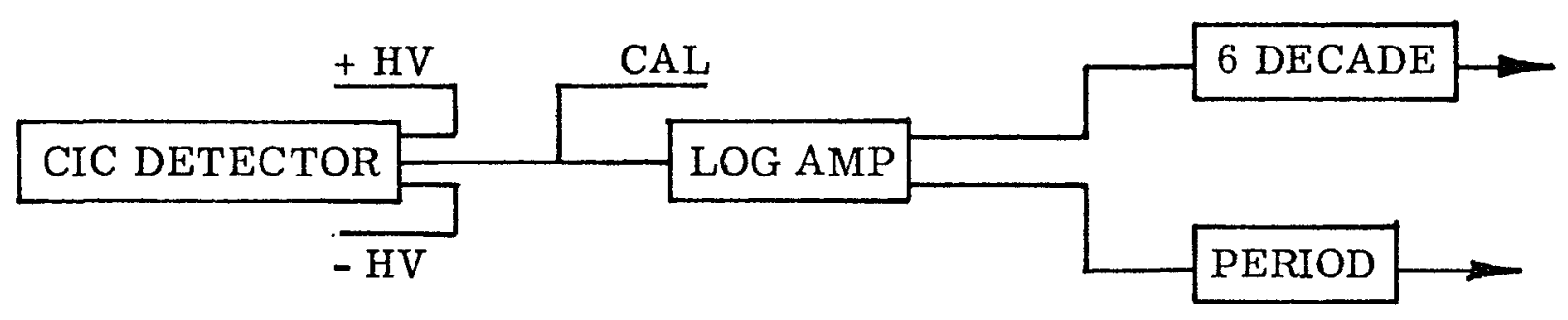

MEDIUM POWER

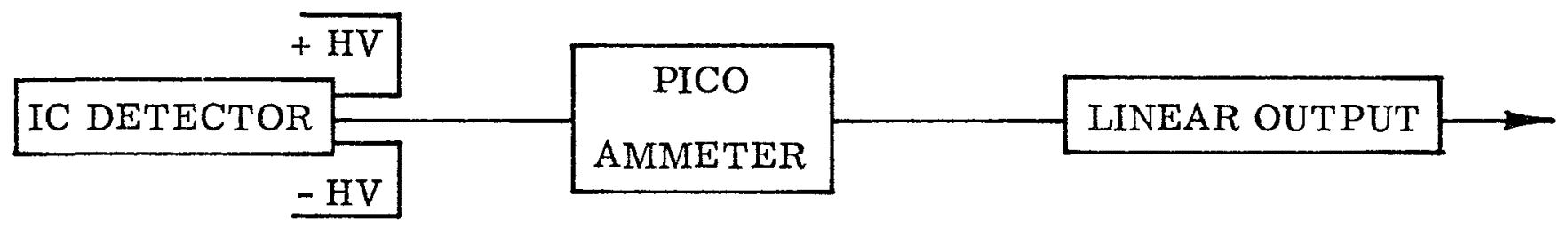

HIGH POWER ,

Figure 3.2.2.2.4

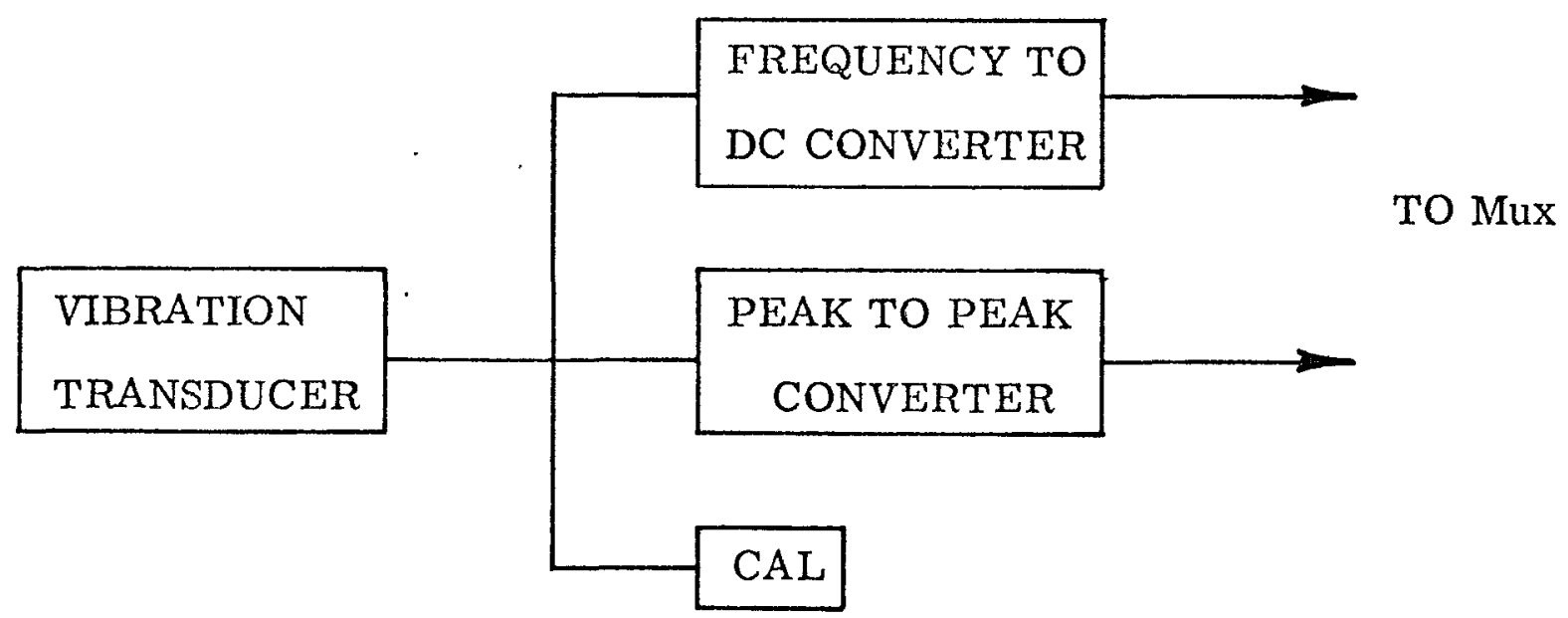

Figure 3.2.2.2.5 
three systems shown in the simplified block diagram are to be used for low power levels and medium and high power levels.

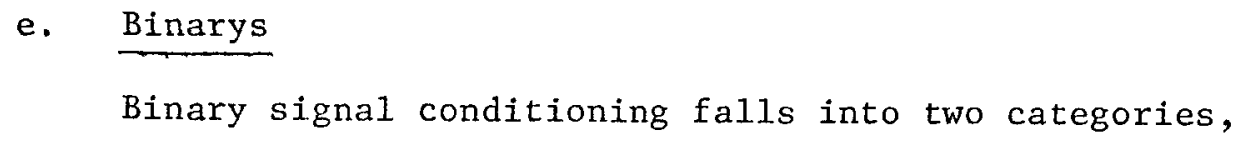

\section{f. Vibration}

Vibration data can be used either in a power spectral density format or converted into its components, amplitude of the envelope and the frequency of the data. The data acquisition system will use the envelope and frequency, as is shown in the block diagram of Figure 3.2.2.2.5. In addition to the peak-to-peak converter and the frequency to DC converter and the vibration sensor, a calibration voltage must be inserted to simulate the vibration envelope and frequency.

\section{g. Speed}

Speed data is acquired from the turbopump as a measure of the number of revolutions per second. This data will be acquired as pulses, 
counted on a per unit time base, stored in a buffer and entered into the computer input in the same manner as the binary data.

\section{h. Positions}

There are three different techniques for measuring positions which may be used: LVDTs, potentiometers, and shaft encoders. The LVDT requires a modulation/demodulation unit, and a calibration source will simulate the potentiometer resistance. Shaft encoder provides a binary signal at its output and requires a power supply and a means of digital calibration.

\section{i. Interface}

The interface with the computer from this portion of the Data Acquisition System will require three basic items: (1) synchronization counter for the speed and binary groupings, (binary) read command and data buffer control, and (3) calibration command input for control of voltage substitutions and other devices providing calibration.

\section{Sampling}

The following paragraphs describe the sampling section of the Data Acquisition System. Consideration should be given to sequential sampling techniques, random sampling techniques, slope and offset correction within the data, sample and hold and the interfaces with the computer and the rest of the Data Acquisition System.

\section{a. Sequence Sampling}

Sequence sampling is performed in a symmetrical manner using a determined order of sampling the data channels. This can be a fixed order 
for the life of the system or it can be generated prior to each frame of data by the computer. A frame of data is defined as the number of samples required to sample each of the highest sample rate channels once. The speed of sampling, that is, the number of samples per second of a given channel, is determined by the number of times per frame that a given channel is sampled. The number of samples per second per channel is in part determined by an error producing process called "aliasing." A mathematical formula is used to determine at what rate grade a given channel should be sampled to reduce the error due to noise and other multiples of the sample rates.

\section{b. Random Sampling}

Random sampling is generated by the computer in response to a demand for data from the control system for data from a given channel. The decision to use random or sequential sampling will be made during the control system analysis program.

\section{c. Slope and offset}

Correction for calibration slope and offset, to provide a standardized output for entrance into the analog to digital coding system, will be provided. The data to be used for slope and offset correction will be generated by calibration of engine measurements and comparison with previous data samples and calibration.

\section{d. Sample and Hold}

Sampled analog data (multiplexed) requires time for switching transients to settle before the data is entered into the analog-todigital conversion seciton. A short time interval of each sampled data channel 
is selected and preserved in a sample and hold network sufficiently long to allow the analog to digital conversion to be completed.

\section{4. $\quad$ Coding}

The last section of the data acquisition system is the section in which the analog data is converted to digital form. Within this section, the analog data is converted to a ten bit binary code with an assigned parity and a sign bit. The data is then entered into the digital computer for use in control calculations, trend data and malfunction detection.

\section{a. Analog to Digital Converter Speed}

The conversion rate of the analog data to digital data

will be determined by the system sampling rate.

\section{b. Word Length}

A ten bit binary code giving $0.1 \%$ resolution, has been selected to satisfy the required accuracy and control bandwidth. An eleventh bit will be used to denote polarity of the signal. A parity bit will be added to the ten bit binary code to determine if the total bit count is odd or even. Odd or even parity will be determined as the programs develop. The total word length is therefore 12 bits.

\section{c. Interface}

There are two interfaces: (1) the sampling and hold synchronization and (2) the computer input of the data that has been encloded and the synchronization pulse back to the computer to encode the next sample. 


\section{DATA CONTROL UNIT}

\section{General}

The primary function of the Data Control Unit is to control instruction data passing from the computer to the Data Acquisition System, Power Distribution Unit and Actuator Drivers and from these three devices to the computer. Raw data from the Data Acquisition System to the computer is transmitted through the Data Control Unit modified sufficiently to match the interface requirements of the computer storage to the Data Acquisition System. Control information coming from the computer to the Data Acquisition System, Power Distribution and Driver Actuator components is decoded within the Data Control Unit. This function, again, performs an interface to prepare control data levels and information into format acceptable to the using devices.

\section{Decode}

The decode section of the Data Control Unit is as shown in the block diagram of Figure 3.2.2,2.6.

\section{a. Receivers}

The function of the receiver is to receive information from the flight computer and condition it for entry into a decoding system.

\section{b. Command Decodes}

The command decoders separate the information into the commands required for the Data Acquisition System, Power Distribution Unit and the Driver Actuator system. Further decoding will probably be required in the user module in order to detail the command to the functions within the modules. The coding and encloding formats will be determined during the preliminary design study phase. 


\section{c. Timing}

The system timing information is generated by the Control Computer. In order to use the timing information within the Data Acquisition System, Power Distribution Unit and the Actuator Drive system, decoding is required. The timing data will arrive at the Data Control Unit from the Control Computer within the same data string as the control information.

\section{Encode}

Figure 3.2.2.2.7 shows the Encode portion of the DCU. Data from the Data Acquisition System to be sent to the computer for storage will require format conversion within the Data Control Unit. After the conversion has been completed, the signals sent to the computer will require an interface medium to provide the transmission power and to match the characteristic impedance of the transmission lines to the Control Computer input.

\section{Interface}

The Data Control Unit will interface with the Control Computer Data Acquisition System, Power Distribution Unit and Actuator Driver components. The electrical physical characteristics will be determined during the design phases.

\section{CONTROL COMPUTER}

\section{Genera1}

The computer required for control of the engine will be a digital computing system capable of operating efficiently in a real time environment. The Control Computer will consist of the major sections as shown in the simplified block diagram of Figure 3.2.2.2.8. 


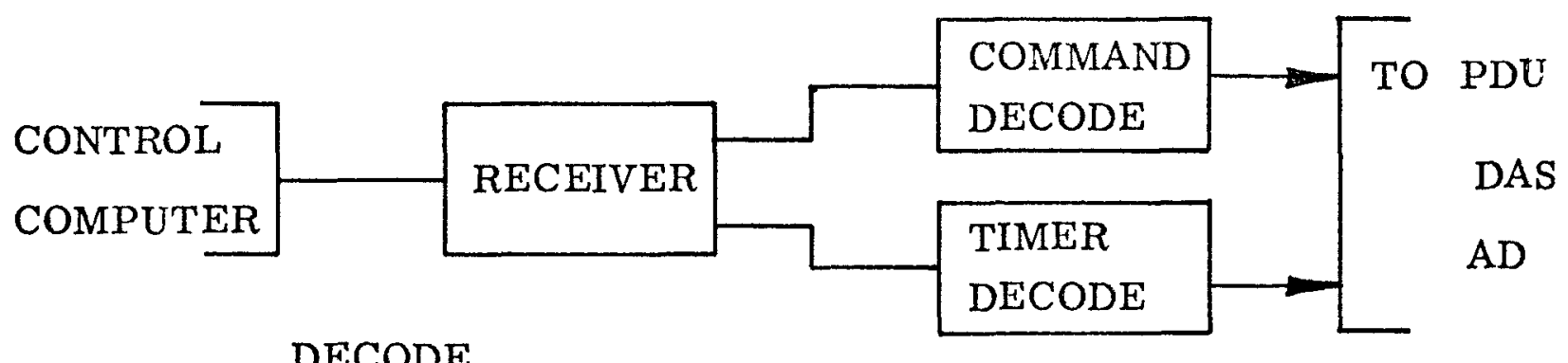

Figure 3.2.2.2.6

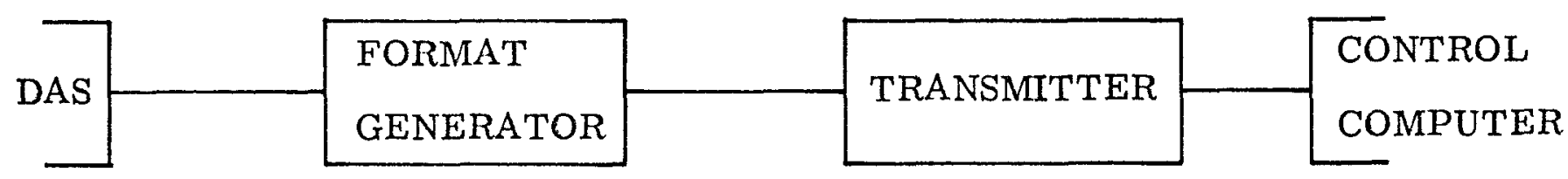

ENCODE

Figure 3.2.2.2.7

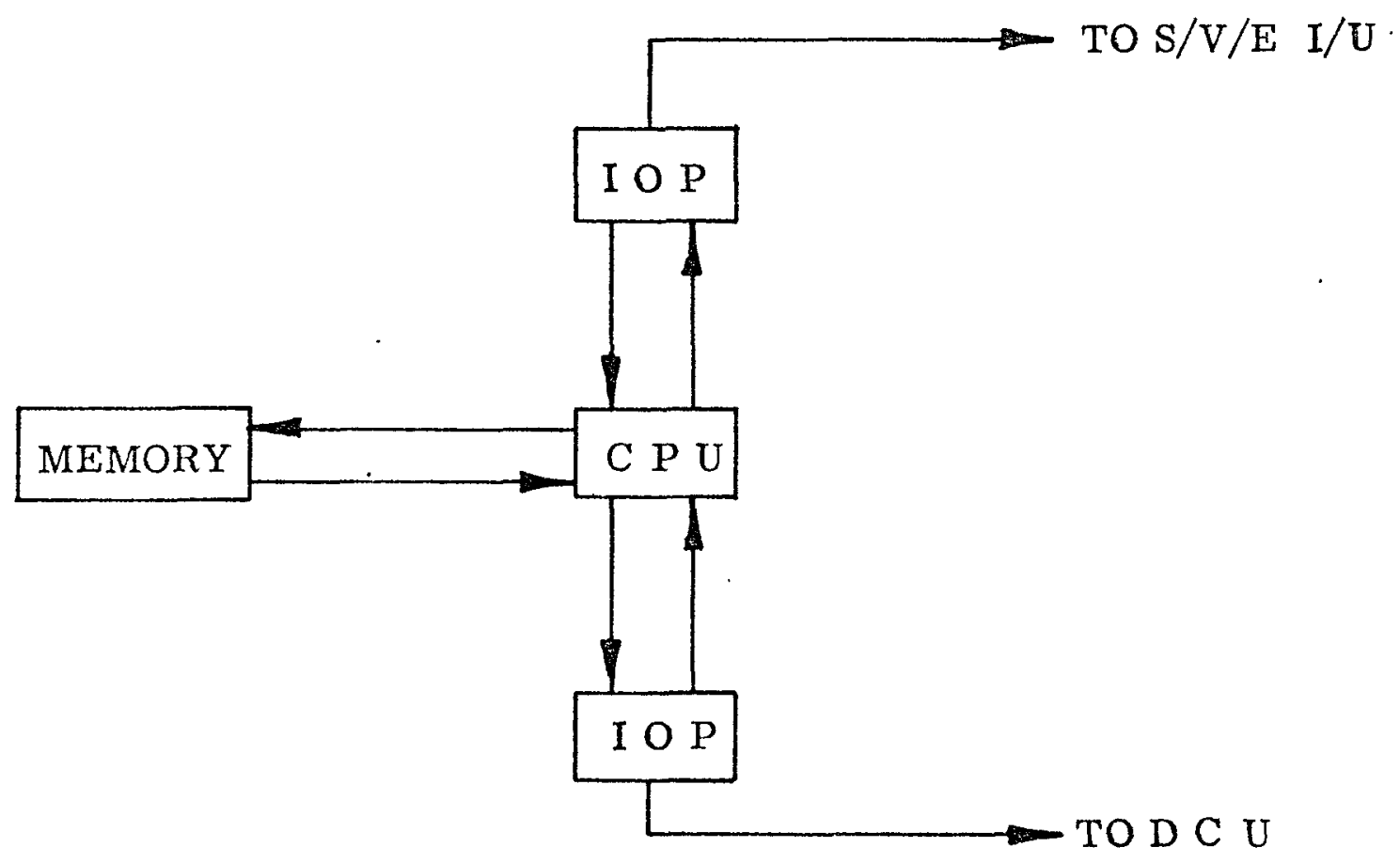

Figure 3.2.2.2.8 


\section{Input/Output Processors (IOPs)}

The computer interface with the remainder of the I\&C system is performed via the input/output processors. The computer system will include automatic input/output processors containing all required data'transfer logic, buffering and signal conditioning to allow data transfers to and from the Data Control Unit, Stage/Vehicle/Engine Interface Unit and memory, inciefendent of the central processor control once a path is established by snftware.

\section{Central Processing Unit}

The Central Processing Unit (CPU) will contain registers, interrupt system, timing system, and the aritmetic unit. It will be capable of addressing memory, performing mathematic logical and shift operations, retrieving and interpreting instructions, processing interrupt and controlling the storage of information.

\section{a. Registers}

The CPU should have multiple registers that can be manipulated under program control. During the preliminary design, a maximum and minimum number of registers required will be determined. The determination will be a function of the speed of processing required, degradation in processing by not having multiple registers, availability of registers for temporary storage and the use of the registers as accumulators for fixed and floating point calculations.

\section{b. Interrupts}

A real time on-line digital control system makes use of a computer using an interrupt system. This is the medium by which a peripheral or control device informs the computer that it needs attention, whether it is data input or a malfunction. To meet this requirement multiple interrupts are 
required to provide several levels of priority within the system. The priorities and post interrupt programs are assigned during the control system software preparation.

\section{c. Timing}

System timing for collection of data and updating the control system will be generated within the timing portion of the central processor. It is here that the timing pulses for use in the Data Control Unit and Data Acquisition System are generated. The timing is also used to synchronize the processes within the Central Processing Unit.

\section{d. Speed}

The number of data bits and words required to be processed per second will be determined during the preliminary design phase. Overall speed will be a function of the number of program steps per second required to control the engine and the amount of intelligence required in each data word of each program step.

\section{Memory}

Until recently, flight computers used magnetic core as the medium to store data and instructions, however, recent developments of other devices are leading to new techniques such as semiconductor memory and magnetic bubbles. The physical medium to be used for data storage will not be determined during prelimianry design; however, the amount of data storage will be determined. Data acquisition studies made during the past year have indicated that in excess 128,000 words of storage will be required. That storage will be required to be available with multiple access paths to memory to prevent slow-down by the input/ output processors requesting simultaneous access to the memories along with the central processor. 


\section{E. STAGE/VEHICLE/ENGINE INTERFACE UNIT}

Stage/Vehicle Applications of NERVA reviewed to date use a data bus concept for communication with major subsystems. The function of the Interface Unit (IU) is to receive instructions from the data bus for use in directing I\&C operations and to send data to the Stage/Vehicle for use in other subsystems. Within the IU a formatting function will be performed to prepare the instructions and data for transmittal to the Control Computer or data bus. This component interfaces with the data bus and Control Computer as shown on the simplified block diagram of Figure 3.2.2.2.9.

\section{F. ACTUATOR DRIVERS}

Actuator Drivers perform the function of providing the electrical signal to the four gimbal actuators and 18 of the valve actuators. The command signal format will be determined during preliminary design; however, the simplified block diagram of Figure 3.2.2.2.10 is valid whether the signal to the actuators is in digital or analog form.

\section{G. POWER DISTRIBUTION UNIT}

\section{General}

The Power Distribution Unit provides two functions; one, power for the engine mounted electronics and two, actuator drivers for the 18 control drums and eight valves. The Data Acquisition System, Data Control Unit, and the remainder of the Actuator Drivers make up the engine mounted electronics. A bus concept will be used to distribute power from the Power Distribution Unit to the using components. The stage 28 volt DC power will be switched to the Data Acquisition System and the Data Control Unit. A battery bus with stage 28 VDC backup will be used to switch power to the valve and 
control drum Actuator Drivers, as well as the drivers themselves.

\section{Bus Concept}

a. Data Acquisition System/Data Contro1 Unit Power Bus

The power bus will use redundant switching to provide

for system reliability within the simplified block diagram (Figure 3.2.2.2.11).

The power output to the Data Acquisition System and Data Control Unit will be controlled through a load selector. The logic for switching loads and source bus will be provided in the block labeled "logic".

\section{b. Battery Bus}

The battery bus will use batteries on line to support the stage power in providing power to the valve and control drum drivers. Under normal and standby operating modes the power from the stage will be used to control the drivers and actuators; however, during high power demand periods, when the actuators are in motion, supplemental power will be provided from the batteries to handle the large increase in power required. In providing power for the actuators in this manner, the use of large conductors to supply power to the Power Distribution Unit from the stage is eliminated. The batteries are changed, on line, continuously to provide a reliable power source when required. Power to the 18 control drum actuators and eight valve actuators is controlled within the Power Distribution unit.

\section{Load Switching Load Selector}

The load selectors are used to provide power to the Data Acquisition System, Data Control Unit, and Actuator Driver during control system power up time. Load selectors are queued for their action by signals from the computer via the Data Control Unit. The signal from the Data Control Unit 


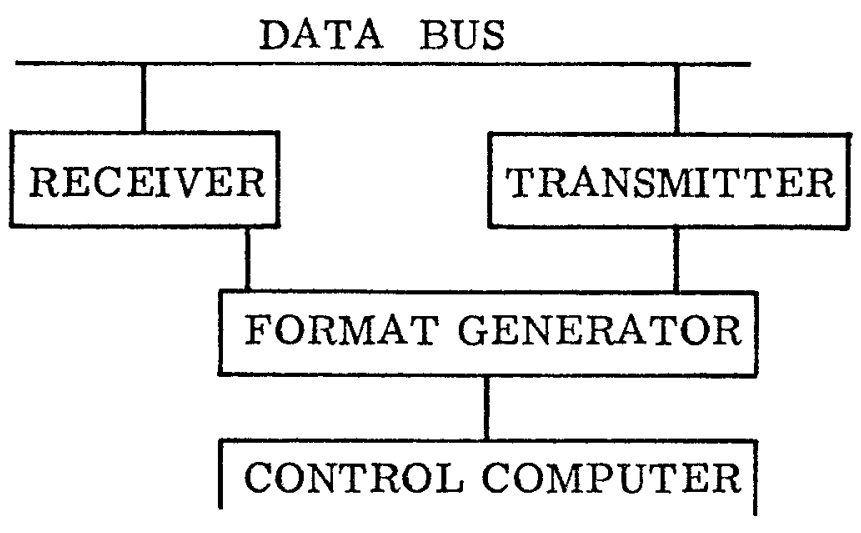

Figure 3.2.2.2.9

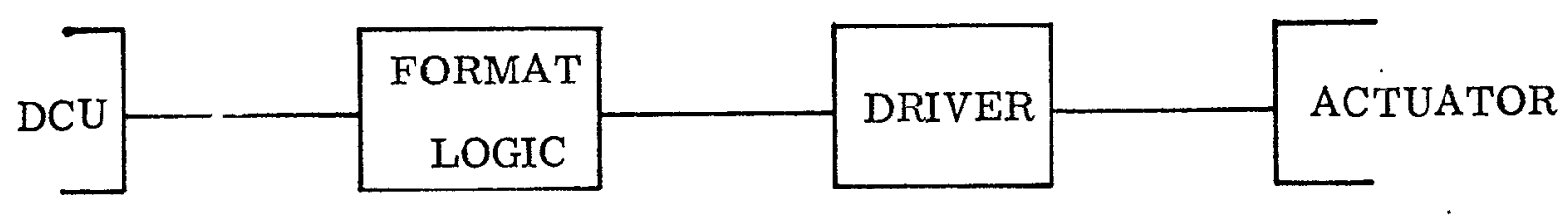

Figure 3.2.2.2.10

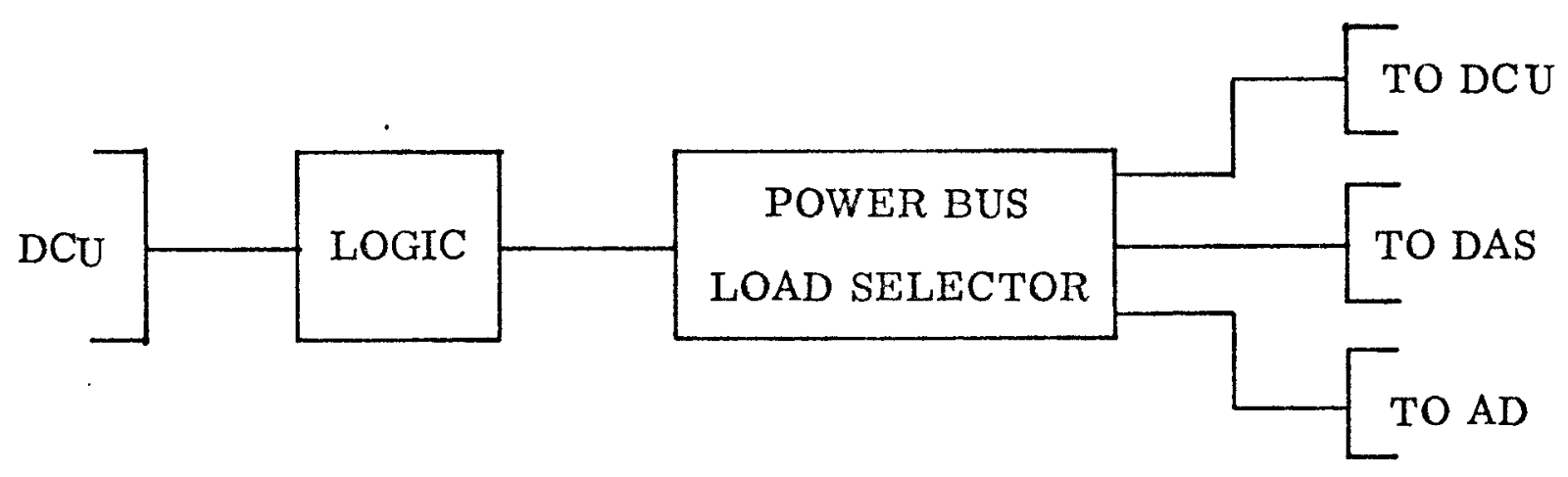

Figure 3.2.2. 2. 11 
is decoded within the Power Distribution Unit and fed to the load selectors via the logic contained within the Power Distribution Unit.

\section{Driver Actuators}

Actuator Drivers incorporated within the Power Distribution Unit receive decoded instructions and output commands to the 18 drum and eight valve actuators. Format of commands and power output required will be determined during preliminary design.

\section{Interfaces}

The Power Distribution Unit shown functionally in Figure 3.2.2.2.11 interfaces with al1 of the engine mounted electronics. The levels of power and the quantitites of redundancy are to be determined during preliminary design.

\section{H. HARNESS ASSEMBLY}

The function of the Harness Assembly is to interconnect all components of the I\&C system acting as the physical interface medium. Preliminary numbers of conductors and connectors have been documented in Reference (a).

\section{SOFTWARE}

\section{General}

The system operational areas requiring software are identified and described below with a discussion of a functional software solution. The software for each of these operational areas is described in functional terms with a specific computer program to be supplied during the software design phase. Software is included as a component because it is the substance used by the engine control system to direct its operational functions. While the software 
does not represent a physically visible component as the other components, software has requirements definition, specifications, design, development and checkout, just as the other components of the I\&C system.

\section{Executive}

The general characteristics of the executive system are listed below. The executive will operate in a real time mode to control the Software component. The simplified block diagram of Figure 3.2.2.2.12 shows the relationship of the executive to the computer system, the engine system, and the applications programs such as data acquisition, calculations and control. It is through the executive that the priority interrupt structure of the control computer is operated. The executive is required to provide an efficient, flexible and reliable system to respond to the control system interrupts and provide the required control system response. There are two major elements of the executive: (1) system supervisory and (2) the input/output monitor. The system supervisor will allocate central processor time on a priority basis such that the highest priority tasks are satisfied in preference to other tasks. The input/output monitor is responsible for processing a11 input/output requests both from the executive and applications programs. The following subsidiary programs are required during the executive operation.

a. A fault system processor that handles system faults and identifies corrective or adaptive procedures to correct the system fault.

b. A resource allocator that will be responsible for allocating memory and managing the priority oriented allocation queue.

c. A timer schedule that will be responsible for monitoring all events that occur on the time ordered basis. 


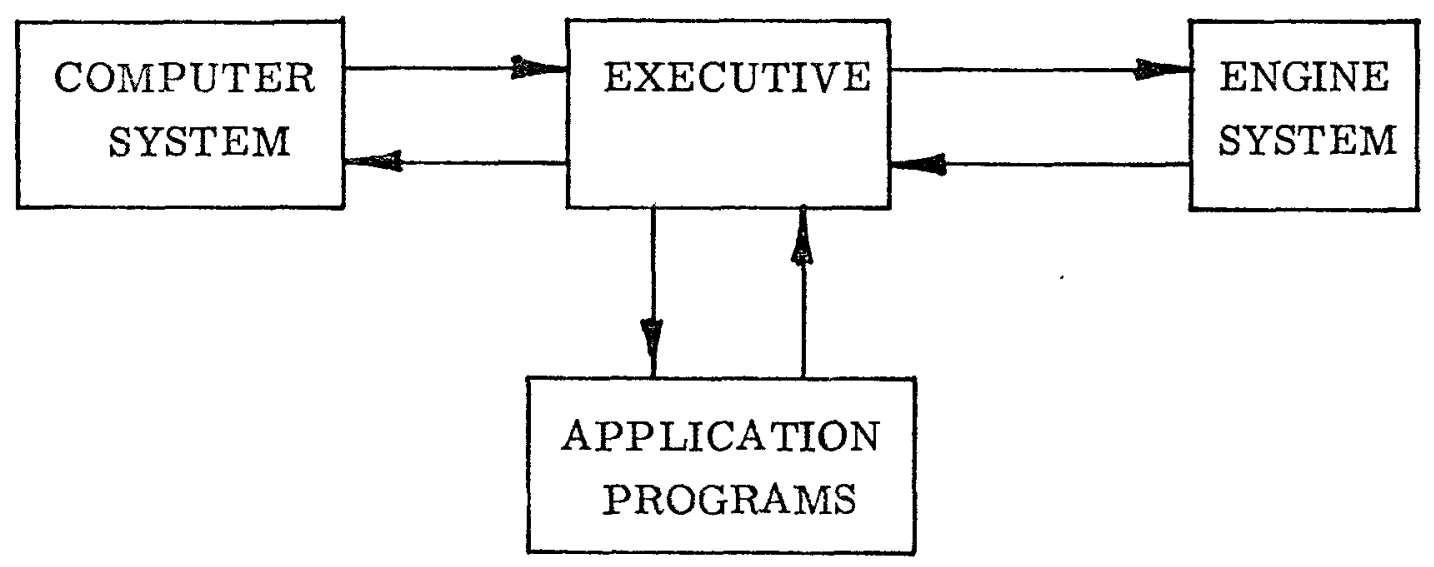

Figure 3.2.2.2.12 
d. A system monitor that will allocate and de-allocate temporary memory space and determine address information for data access.

e. An interrupt handler that processes the various types of interrupts such as external input/output operator communications, timing application programs, error corrections, critical malfunctions, and trend inputs.

\section{Data Acquisition}

Data acquisition software will be divided into two sections; controlling the multiplexer and analog to digital converter, and controlling the data acquisition system calibration routines.

\section{a. Multiplexer Analog to Digital Conversion Control}

Data channel sampling rates and address selection will be determined by the computer and control requirements and will be generated in accordance with the data acquisition software. If the data acquisition preliminary design reveals that a fixed sample rate technique is to be used, then the multiplexer program will be generated to provide maximum data sampling rate required for any foreseeable emergency and at sufficient rate to eliminate any problem with aliasing errors. The multiplexer control software using this technique will require the generation of channel addresses for each channel to be sampled during a frame. (A frame of data is defined as the time interval required to sample each of the channels with the highest sample rate once). If multiplexer control is to be on an adaptive basis, that is, the control system requirements dictate when selected channels are to be sampled, the software will be required to generate the address of the channel to be sampled.

\section{b. Calibration}

Calibrations will be initiated on a periodic basis by the Data Acquisition Systen software. Selected channels will be requested 
to enter a calibration mode to provide calibration data. These data points will be checked by the software by comparing with previous calibration data; in the event that a difference occurs a new correction factor will be generated for use in conversion of raw data. During the calibration process the software will inform the control system calculation routines that the data is in a calibration mode and is not valid for updating the control system.

\section{Calculations}

The calculation program will consist of a series of calculation routines that are required for the solution of control system equations. The routines will be initiated by interrupts at specified intervals. The solutions to the calculations will be placed in a file to be accessed as required by the control software. For calculations involving multiple measurements an averaging will be performed by software before the data is entered in file for control use.

\section{Controls}

The control program will consist of many controls routines and control functions of modular design that will be controlled by a transfer linkage routine. The basic functional requirements of the control software are listed below.

\section{a. Prestart Functional Checkout \\ Prestart functional checkout will consist of providing} test input to all engine components except those which would cause an undesirable change in engine behavior and, after providing this input, monitoring component response. 
b. Startup Contro1

Startup operation will consist of controlling the engine during bootstrap following prescribed impulse time contour.

c. Nórma1 Operating Range Contro1

Normal operating range profile will contain software

for the following characteristics.

(1) Ramp to Design Thrust Along High Isp Contour

(2) Hold at Design Thrust

(3) Throttle

\section{d. Shutdown}

Shutdown profile consists of ramping the engine from

throttle point to cooldown.

\section{e. Cooldown Contro1}

The cooldown period used to remove nuclear core after heat using propellant flow.

\section{f. Malfunction Analysis}

Engine functions that are critical to engine operation must be monitored at a high relative sample rate because of the immediate correction action required. The presence of a malfunction must be passed to the control computer and correction routines initiated at a high rate. The control software will be responsible for adapting the control algorithms to the new conditions brought about by the malfunction. 
g. Trend Data Analysis

Trend data analysis monitors engine functions to determine changes or degradation in engine components. This analysis will be required for long duration application and will consist of a comparison and trial routine. Control function routines will be initiated when trend data software indicates action is required.

\section{Outside Communications}

Software will be required to communicate with the stage and vehicle computers, both to receive command data and to send information to the astronauts or to the stage/vehicle computer files. Software will be required to interpret the stage/vehicle commands and to generate commands in return, to the various functional parts of the I\&C system.

\section{System Check}

This program will provide a means to perform computer selfchecking as well as operational checking the I\&C system. System checking criteria will be established in the areas of engineering unit data comparison, calculated data comparison, event occurrences, program operation, interval timing, diagnostic, check summing, feedback response time, and data verification.

\section{Simulation}

Simulation software, if proven to be desirable, will be required for reactor and engine functions associated with astronaut operation. Simulation models will consist of:

$$
\begin{aligned}
& \text { a. Prestart Functional Checkout } \\
& \text { b. Startup } \\
& \text { c. Normal Operating Range } \\
& \text { d. Shutdown }
\end{aligned}
$$




\section{e. Cooldown \\ f. Malfunction}

\subsection{ENGINE POWER REQUIREMENTS}

The I\&C system has been designed with a self contained battery. This battery is designed to supply the total energy requirements of an engine operation through early cooldown and then to be recharged by the stage. The battery saves cable weight two ways: (1) by eliminating the requirement for the stage to supply the peak-loads that the engine demands; (2) by reducing the steady state stage demand of nearly $2 \mathrm{KW}$ during engine operation, to the 560 watt leve1 during recharge.

The design points for this system are as follows:

1. No stage energy is required during checkout.

2. No stage energy is required during engine operation.

3. No stage energy is required during early cooldown.

4. 560 watts of stage power are required for the period after 1.1 and up to 4.6 hours of pulse cooldown depending on mission and burn. (This is the period when the battery is recharged.)

5. 80 watts of stage power are required for the remainder of cooldown (after 4.6 hours) up to 24 hours.

6. 180 watts of stage power are required for the remainder of cooldown after 24 hours. (A 100 watt trickle charge of the battery is started after a 24 hour stand time).

7. 130 watts of stage power are required during coast.

8. 130 watts of stage power are required in the docked condition.

Figure 3.3.1 graphical1y depicts the actual engine demands and Figure 3.3.2 depicts the stage demands. 


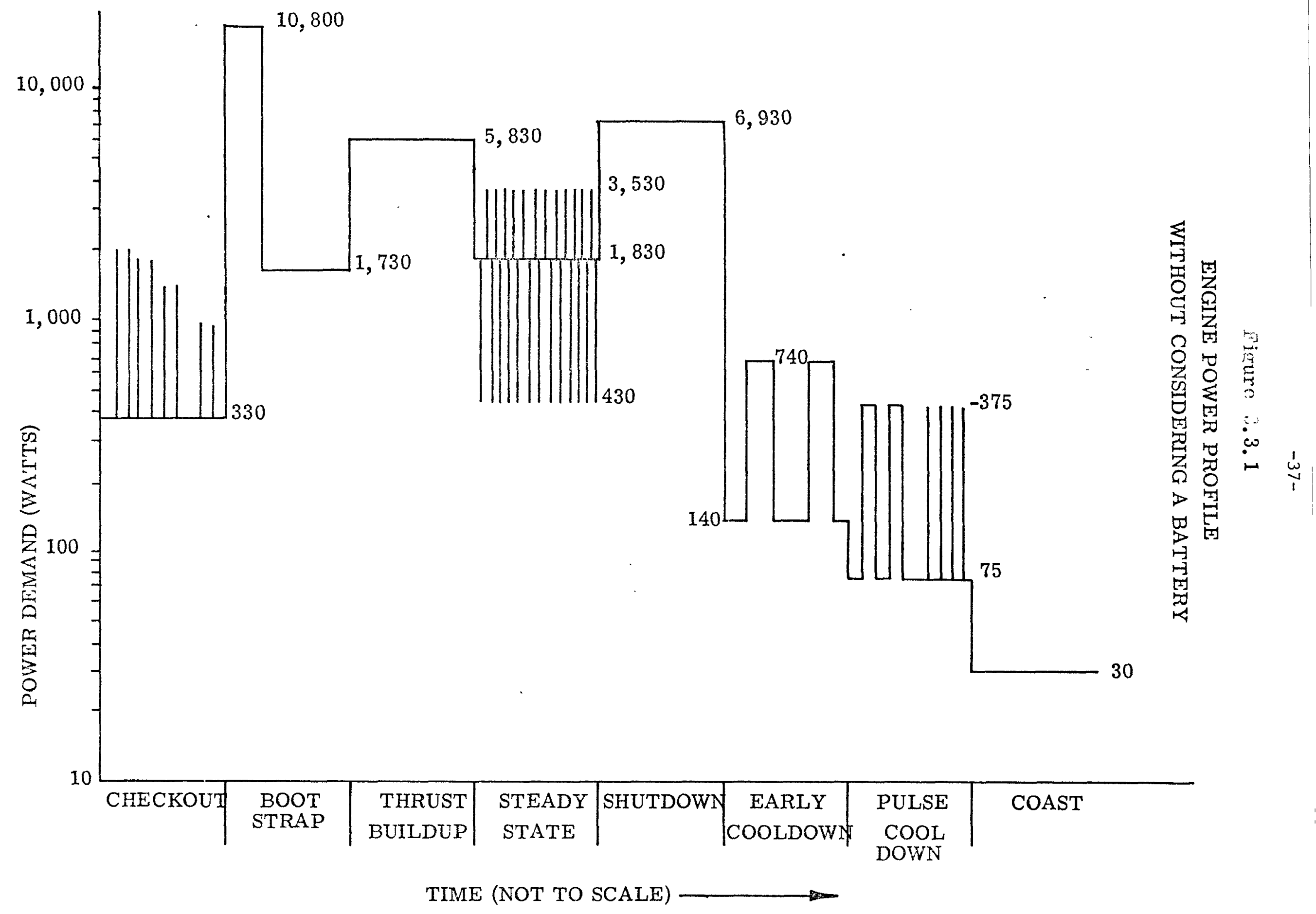




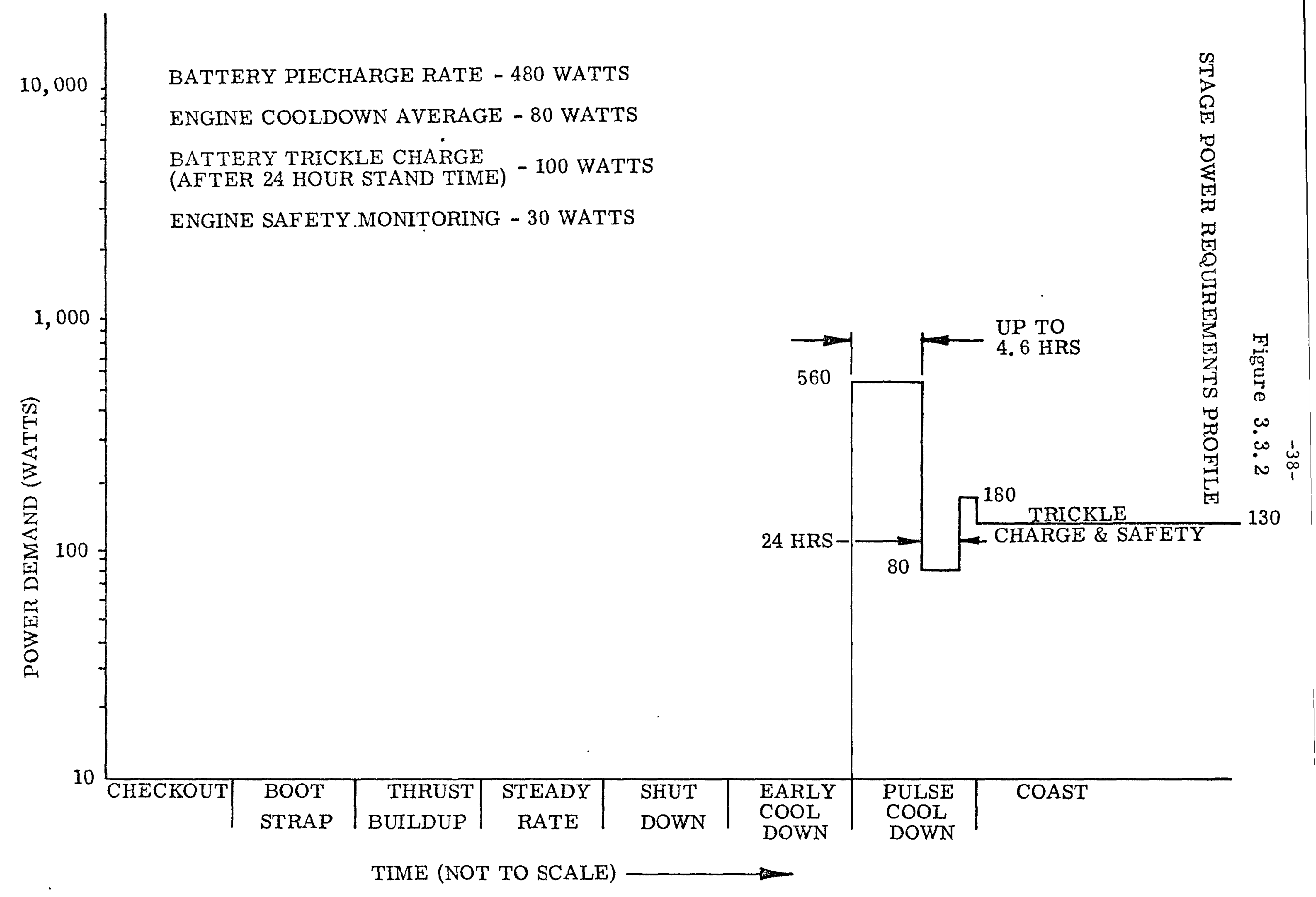




\subsection{DESIGN REQUIREMENTS}

To the present time, only four of the nine I\&C system components were actively worked. These were the Data Acquisition System (DAS), Data Control Limit (DCL), Power Distribution Unit (PDU) and the Actuator Driver Unit (ADU). Preliminary Design Sheets were prepared for each of these components and are included in Appendix A through D. Preliminary Block Diagrams for each component were prepared and are included in Appendix $\mathrm{E}$.

\subsection{ELECTRICAL INTERFACE DRAWINGS}

Work in preparing preliminary electrical interface drawings had progressed to the point where they were released as ANSC Drawing 1139949 NERVA I\&C Subsystem Flight Interface Networks. This drawing with its many sheets would have provided the basis for the engine electrical system interconnection.

\subsection{RELTABILITY}

Work had not progressed far enough to evaluate the reliability of the designs documented in the Appendices. The design is sufficiently mature to permit predicting that the system was feasible and could possibly be made to meet the reliability requirements. 
REFERENCES

A. Propulsion Module Study - Engineering Operations Report N8610R:72-029, March 1972

B. Performance Design \& Qualification Requirements for Instrumentation and Control System EC 90214A, Apri1 1971

C. Preliminary Measurement Requirements List for the NERVA Operational Flight Engine 1137400E-C101-CP090290-F1, October 1971

D. Memo N7820:3141N, C. W. Hennige to E. V. Krivanec, 28 August 1970, Reference Engine Multiplexer Description. 


\section{DATA ACQUISITION SYSTEM}

\section{DESIGN SHEET}

\section{$1.0 \quad \underline{S C O P E}$}

This design sheet establishes the performance, test and qualification requirements for that portion of the I\&C System designated as the Data Acquisition system. The function of the Data Acquisition System (DAS) is to condition the output of engine sensors to produce a digital representation of engine measurements for use in engine control system calculations.

\section{$2.0 \quad$ APPLICABLE DOCUMENTS}

TBD

\section{$3.0 \quad$ REQUIREMENTS}

3.1 Performance - The DAS shall be capable of meeting the performance requirements specified herein.

3.1.1 Functional Characteristics - The DAS shall provide excitation to passive engine sensors and accept raw data from passive and active engine sensors and from other engine control system components. It shall accept commands and instructions from, and provide engine and control system data to the Data Control Unit.

3.1.1.1 Primary Performance Characteristics - The DAS shall process the input described in 3.1 .1 to provide digital data for control, trend and malfunction calculations.

\subsection{Conditioning - Excitation shall be provided for the} following types of sensors:
a. Strain Gage
b. Eddy Current
c. Potentiometrics
d. Neutronic
e. Resistance Temperature Transducer 


\subsection{Calibration - Calibration for each measurement} channel shall be provided by shunting and/or substitution processes.

\subsection{1 Control - Calibration switching shal1 be con-} trolled by commands received from the Data Control Unit and decoded within the Data Acquisition System.

\subsection{2 Amplification/Filtering - Each type of channel} shall be filtered as necessary to eliminate aliasing errors. If filtering is required, the engine sensor output shall be buffered to isolate the filter then the sensor. An amplifier shall be used to provide a voltage gain if required.

3.1.1.2 Multiplexing - A1l analog data channels shall be sampled on a time ordered sequence. Time interval between samples per channel shall be determined by control system requirements for updated measurements. Channel number identification shall be provided by a sampling sequence list generated by the control computer and decoded within the Data Acquisition System.

\subsection{Channel Sequencing - The sequence of data channel} multiplexing shall proceed according to the structure of a data frame. A frame of data is defined as the number of channels required to sample each of the highest sample rate channels once. Capability shall also be provided for sampling selected channels on a random basis as required by control calculations and adaptive functions of the engine controller.

3.1.1.1.2.2 Signal Leve1 - The level of the signals entering the multiplexer are TBD.

3.1.1.1.2.3 Sample and Hold - A sample and hold amplifier shall be used at the output of the multiplexer with an aperture TBD. The average signal level during the aperture shall be held long enough to provide a source for analog to digital conversion of individual channels. 
3.1.1.1.3 Analog to Digital Conversion - Each analog data input channel shall be converted to a digital code consisting of 10 binary data bits full scale plus a sign bit. A parity code shall be generated within the analog to digital converter.

3.1.1.1.3.1 Control - The analog to digital (A/D) converter shall be a free-running device synchronized by the system time code generator that will convert the output of the sample and hold amplifier to the format of 3.1.1.1.3. The A/D converted output shall load the Data Acquisition System output buffer on command of the Data Acquisition System Decode instruction.

3.1.1.1.4 Binary Input Data - Data generated in digital form such as a shaft encoder or a switch closer indicating limit travel of a valve shall be treated as binary data.

3.1.1.1.4.1 Conditioning - Conditioning of binary data shall consist of pulse shaping and grouping into words similar to those generated by the analog to digital converter in the analog portion of the Data Acquisition System.

3.1.1.1.4.1.1 Switch Position Data - Switch position data shall be collected in a buffer that will generate a data word containing information on five devices. Each device shall have two bits within the data word; one for the extent of travel of a device in each of the full extreme positions.

3.1.1.1.4.1.2 Digital Inputs - The inputs from devices generating a digital word such as a shaft encoder shall be transferred directly to the serial to parallel buffer, from the binary signal conditioning input element.

3.1.1.1.4.2 Intermediate Buffer - The intermediate buffer sha11 hold the digital input data in short term temporary storage waiting space within the output buffer. The intermediate buffer shall be controlled by signal command generated within the Data Acquisition System instruction decoder. 
3.1.1.1.5 Output Buffer - The output buffer shal1 organize the Data Acquisition System output sequence by integrating the digitized analog data inputs and the digital data input into a sequence of data to be sent to the computer. The output buffer shall receive its instructions from the Acquisition System Instruction decoder.

3.1.1.1.6 Instruction Decoder - The instruction decoder element in the Data Acquisition System shall receive commands from the Data Control Unit and generate instructions for signal conditioning multiplex, analog to digital conversion, digital input intermediate buffer and the Data Acquisition System output buffer elements.

3.1.1.1.6.1 Signal Conditioning - Instructions to the signal conditioning element shall contain calibration commands, both shunt and substitution and provide data required for calibration factors.

3.1.1.1.6.2 Multiplex - Multiplex instructions shall instruct the multiplexer to sample each input data channel in the current frame sequence. Instructions shall contain data for gain change and offset within the multiplexer amplifier.

3.1.1.1.6.3 A to D Conversion - Instructions to the A to $D$ converter shall contain synchronization bits for the sample and hold amplifier and $A$ to $D$ conversion.

3.1.1.1.6.4 Intermediate Buffer - Instructions to the intermediate buffer shall shift its contents to the Data Acquisition System output buffer.

3.1.1.1.6.5 Data Acquisition System Output Buffer - Instructions to the Data Acquisition System output buffer shall shift its contents out of the Data Acquisition System and into the Data Control Unit. 


\section{DESIGN SHEET}

\section{$1.0 \quad \underline{S C O P E}$}

This design sheet establishes the performance, test and qualification requirements for that portion of the I\&C System designated as the Data Control Unit. The function of the Data Control Unit (DCU) is to format raw data from the Data Acquisition System for Control Computer use and to decode commands/ instructions from the Control Computer for use by other I\&C components.

$2.0 \quad$ APPLICABLE DOCUMENTS

TBD

\subsection{REQUIREMENTS}

3.1 Performance - The DCU shall be capable of meeting the performance requirements specified herein.

3.1.1 Functional Characteristics - The DCU has two functions. One is to group data from the Data Acquisition System in computer format and transmit that data to the Control Computer and, two, to interpret commands and instructions from the Control Computer and generate instructions for the Data Acquisition System, Power Distribution Unit and Actuator Drivers. The Data Control Unit also provides time signal and clock rates for the I\&C system timing and event sequencing.

\subsubsection{Primary Performance Characteristics - There are two} primary performance characteristics: (1) data transmission, and (2) command/ instruction decode and transmission.

3.1.1.1.1 Data Transmission - Data shal1 be received from the Data Acquisition System in binary form converted to computer compatible format and groups to include other required data and transmitted on command to the control Computer. The following elements are required to format the data and to transmit it to the Control Computer. 
1. Input Buffer

2. Computer Format Generator

3. Output Buffer

4. Parallel to Serial Converter

5. Line Driver Amplifier

\subsection{Input Buffer - The input buffer shall receive the} raw data stream from the Data Acquisition System, check the parity of each data word add identification information and hold the data word until the computer format generator demands a new input. Data sha11 be shifted in and out of the input buffer by a time sequence code provided by the system time and sequence generator.

\subsection{Computer Format Generator - The computer format} generator sha1l convert the data to Control Computer format. A time code indicating chronology of data samples shall be added. Each data work assembled shall be shifted to the output buffer on command of the system time code and sequence generator.

3.1.1.1.1.3 Output Buffer - The output buffer shall receive the output of the computer format generator and hold the computer word until the parallel to serial converter is ready to receive the next computer word. Shifting of data in and out of the buffer shall be controlled by system time code sequence generator.

\subsection{Parallel to Serial Converter - The parallel to serial} converter shall accept data words from the output buffer and convert the parallel word from the output buffer form that is output to the line driver amplifier. The output serial bit rate is controlled by the system time code sequence generator.

\subsection{Line Driver Amplifier - The line driver amplifier sha11} receive the serial bit stream from the parallel to serial converter and amplify each bit to provide sufficient power to drive a transmission line from the engine mounted electronics to the Control Computer above the propellant tank. Amplification shall provide sufficient pre-emphasis to climinate distortion in the transmission line and provide a usable pulse at the input to the Control Computer. 


\subsection{Contro1 Computer Command/Instruction Decode - The} computer command/instruction decode unit sha11 receive and decode command/ instructions from the Control Computer into specific instructions to components of the I\&C system and for elements of the Data Control Unit. The command/instruction decode generator sha1l also decode the system time signal received from the Control Computer into usable time codes and distribute them to the using components of the I\&C system and the elements of the Data Control Unit. The computer command/instruction decode unit contains the following elements:

$$
\begin{aligned}
& \text { 1. Line Received } \\
& \text { 2. Command/Instruction Decoder } \\
& \text { 3. Actuator Driver Instruction Formatter } \\
& \text { 4. Power Distribution Unit Instruction Formatter } \\
& \text { 5. Data Acquisition System Instruction Formatter } \\
& \text { 6. System Time Code Generator }
\end{aligned}
$$

\subsection{Line Receiver - The line receiver shall receive} commands/instructions and timing data from the Control Computer. It shall provide Control Computer transmission line termination. It shall amplify and condition the data received from the Control Computer such that the output of the line receiver can drive the input to the system decoder.

$$
\text { 3.1.1.1.2.2 System Decoder - The system decoder shall decode }
$$
and separate the instruction for controlling the following: Data Acquisition System, Power Distribution Unit, Actuator Drivers and System Time Code Generator.

\subsection{Data Acquisition System Instruction Formatter - This} element shall receive instructions from the system decoder and prepare instructions for the Data Acquisition System that controls calibration of multiplexer, analog to digital conversion, and buffer control instructions.

\subsection{Power Distribution Unit Instruction Formatter - This} unit sha11 receive instructions from the system decoder and prepare commands to control power bus switching within the Power Distribution Unit. 


\subsection{Actuator Driver Instruction Formatter - This item} shall receive instructions from the system decoder, separate the instructions into data for each actuator driver and transmit the data to each actuator driver. The output code shall refresh the actuator drivers at a rate TBD to provide instructions to the actuator at an update rate to accommodate any malfunctions or normal control action.

\subsection{System Time Code and Sequence Generator - The system} time and sequence generator shall accept a coded time message from the system decoder. The time code received from the system code the time code generator shall be used to generate timing and sequence codes for use in the Data Acquisition System and the Data Control Unit as indicated in Paragraphs 3.1.1.1.1. 


\section{DESIGN SHEET}

\section{POWER DISTRIBUTION UNIT}

\section{$1.0 \quad$ SCOPE}

This design sheet establishes the performance, design, test and qualification requirements for that portion of the I\&C System designated as the Power Distribution Unit. The function of the Power Distribution Unit (PDU) is to supply electrical energy to meet all the requirements of all parts of the engine.

\subsection{APPLICABLE DOCUMENTS}

TBD

\subsection{REQUIREMENTS}

3.1 Performance - The PDU shall be capable of meeting the performance requirements specified herein.

3.1.1 Functional Characteristics - The PDU shall be capable of performing the operational functions specified herein.

\subsubsection{Primary Performance Characteristics}

3.1.1.1.1 Use of Stage Power - The PDU shall demand energy from the stage only during pulse cooldown, coast and docked periods. At all other times the PDU shall meet all engine power requirements with self-contained batteries.

3.1.1.1.1.1 Limits on Stage Power Demands - The PDU demands on the stage power supply shall not exceed the following:
a. $\quad 560$ watts during pulse cooldown
b. $\quad 130$ watts during coast
c. 130 watts during docked condition

3.1.1.1.1.2 Power Input Buses - The PDU shall be designed to operate from either of two separate input buses with isolated negative returns. 
3.1.1.1.2 Batteries - The PDU sha11 use two rechargeable batteries connected to separate power buses each of which shall be able to furnish the needs of the engine during checkout, operation and early cooldown.

3.1.1.1.2.1 Capacity - Each battery shall be capable of supplying the total engine requirements during these periods while being discharged to no more than $50 \%$ of its capacity during normal mode operation.

3.1.1.1.2.2 Battery Recharge - Each battery shall be capable of being independently and, upon command, recharged at a C/5 rate. Charge control circuitry shall be used to determine stage of charge and terminate charging when batteries are returned to $100 \%$ of capacity.

3.1.1.1.2.3 Battery Switching - Each battery shall be capable, on command, of being switched to the alternate bus.

3.1.1.1.3 Power Buses - Two power buses shall be used, shall be independent and shall have isolated negative returns. Each user of power shall be able to satisfy its demands from either bus.

3.1.1.1.3.1 Power Bus Voltage Regulation - Each bus shall be maintained within the following limits during all phases of engine operation:

$$
\begin{aligned}
& \text { a. Steady state: } \pm \text { TBD volts } \\
& \text { b. Transient: TBD }
\end{aligned}
$$

3.1.1.1.3.2 Power Bus Loading - Peak instantaneous, and average power demands made of the power buses by engine components shall not exceed the values listed in Tables 1 through TBD.

3.1.1.1.3.3 Power Bus Load Limiting - Provision shall be made to limit the steady demands made of each bus to $120 \%$ of the maximum steady state values listed in Tables 1 through TBD. In the event that a load exceeds $120 \%$ of maximum it shall be disconnected from the bus.

3.1.1.1.4 PDU Status - Measurements shall be made to determine the stateof-health of the PDU. These measurements shall be available to the Data Acquisition system (DAS) on demand. 
3.1.1.1.4.1 Analog Status Measurcments - These measurements shall have an output range of 0 to TBD volts with a source impedance of TBD ohms or less and a frequency content of less than $1 \%$ of full scale above TBD Hz. The following measurements sha11 be made:

a. Battery state-of-charge
b. Stage input voltage
c. Battery current
d. Bus current
e. Bus voltage
f. TBD

3.1.1.1.4.2 Binary Status Measurements - These measurements shall be combined into eleven bit plus parity digital words compatible with the DAS digital data format requirements and sha1l be transmitted to the DAS upon receipt of interrogate commands. The following measurements shall be made:
a. Charge battery 1
b. Charge battery 2
c. Charge battery 1 to bus 2
d. Charge battery 2 to bus 1
e. Interrogate binary status
f. TBD

\subsection{Command Format - TBD}

\subsection{Stage Use of PDU Energy - During some docking and mating} operations the stage may require power from the PDU batteries. The power required shall be limited to TBD watts peak and TBD watt hours maximum. After use of this energy the stage shall supply sufficient energy to permit the PDU to recharge the batteries prior to engine operation.

\subsubsection{Secondary Performance Characteristics - There are no secondary} performance characteristics.

3.1.1.3 through $6.0-$ TBD 


\section{ACTUATOR DRIVER UNIT}

\section{DESIGN SHEET}

\section{$1.0 \quad \underline{S C O P E}$}

This design sheet establishes the performance, design, test and qualification requirements for that portion of the I\&C system designated as the Actuator Driver Unit. The function of the Actuator Driver Unit (ADU) is to accept commands from the Data Control Unit and translate them via power conditioning, switching and/ or amplification into the form required to operate all of the engine actuators.

\subsection{APPLICABLE DOCUMENTS}

TBD

\subsection{REQUIREMENTS}

3.1 Performance - The ADU shall be capable of meeting the performance requirements specified herein.

3.1.1 Functional Characteristics - The ADU shall be capable of performing the operational functions specified herein.

\subsubsection{Primary Performance Characteristics}

3.1.1.1.1 Input Power - Input power to the ADU will be held within the following limits:

$$
\begin{aligned}
& \text { a. Steady State: } 28 \mathrm{~V} \pm \text { TBD volts } \\
& \text { b. Transient: TBD }
\end{aligned}
$$

3.1.1.1.1.1 Input Buses - The ADU shall be designed to operate from either of two separate sources which carry isolated returns.

3.1.1.1.1.2 Power Consumption - The ADU shall consume less than TBD watts peak during engine operation Consumption during coast shall be less than TBD watts.

3.1.1.1.2 Command Input - Commands are divided into two classes: discrete and demand. All commands will be digital in nature with TBD \pm TBD volts DC indicating a binary " 1 " and TBD \pm TBD volts DC indicating a binary " 0 ". 


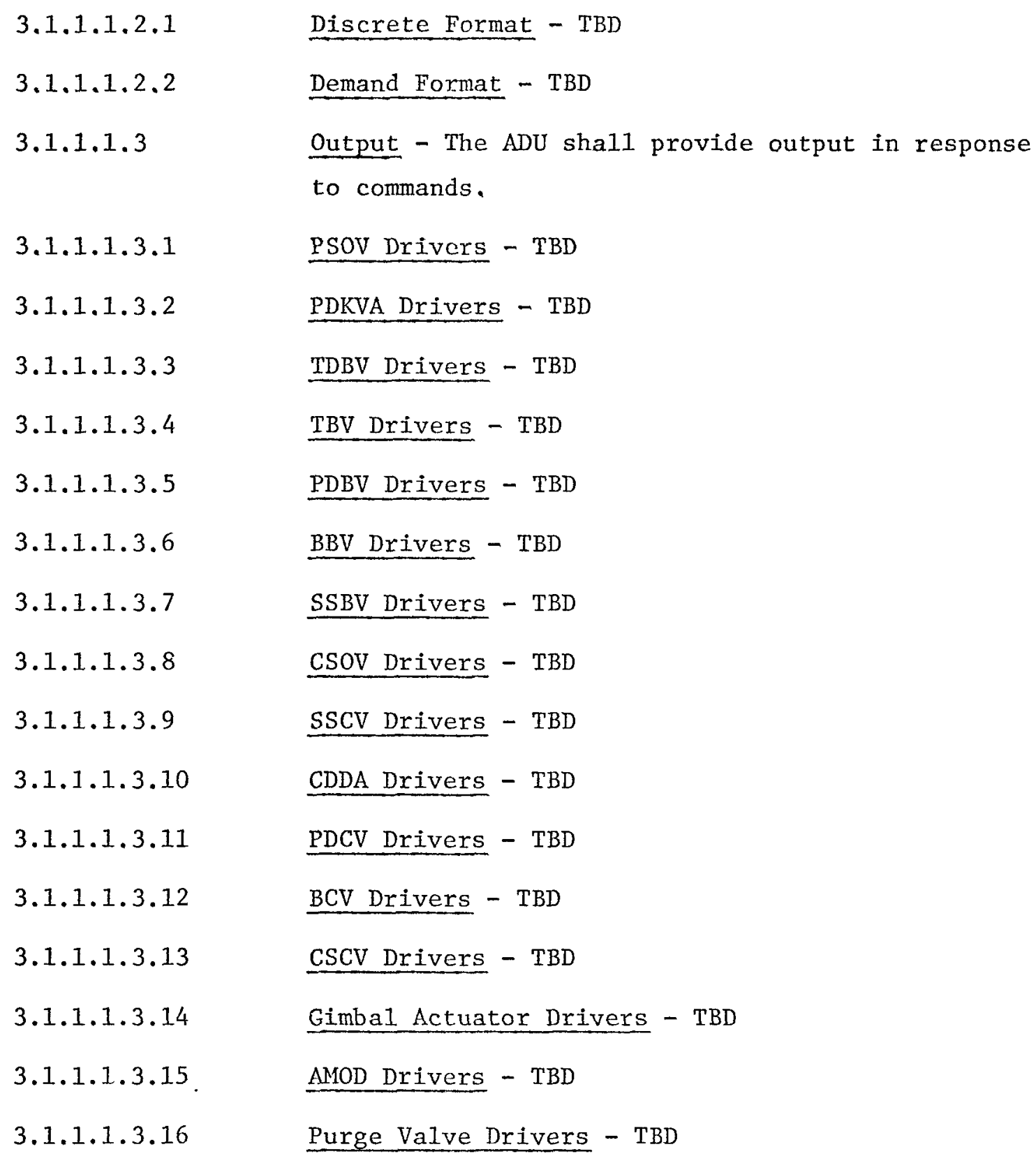


APPENDIX E

I\&C SYSTEM COMPONENT FUNCTIONAL BLOCK DIAGRAMS 


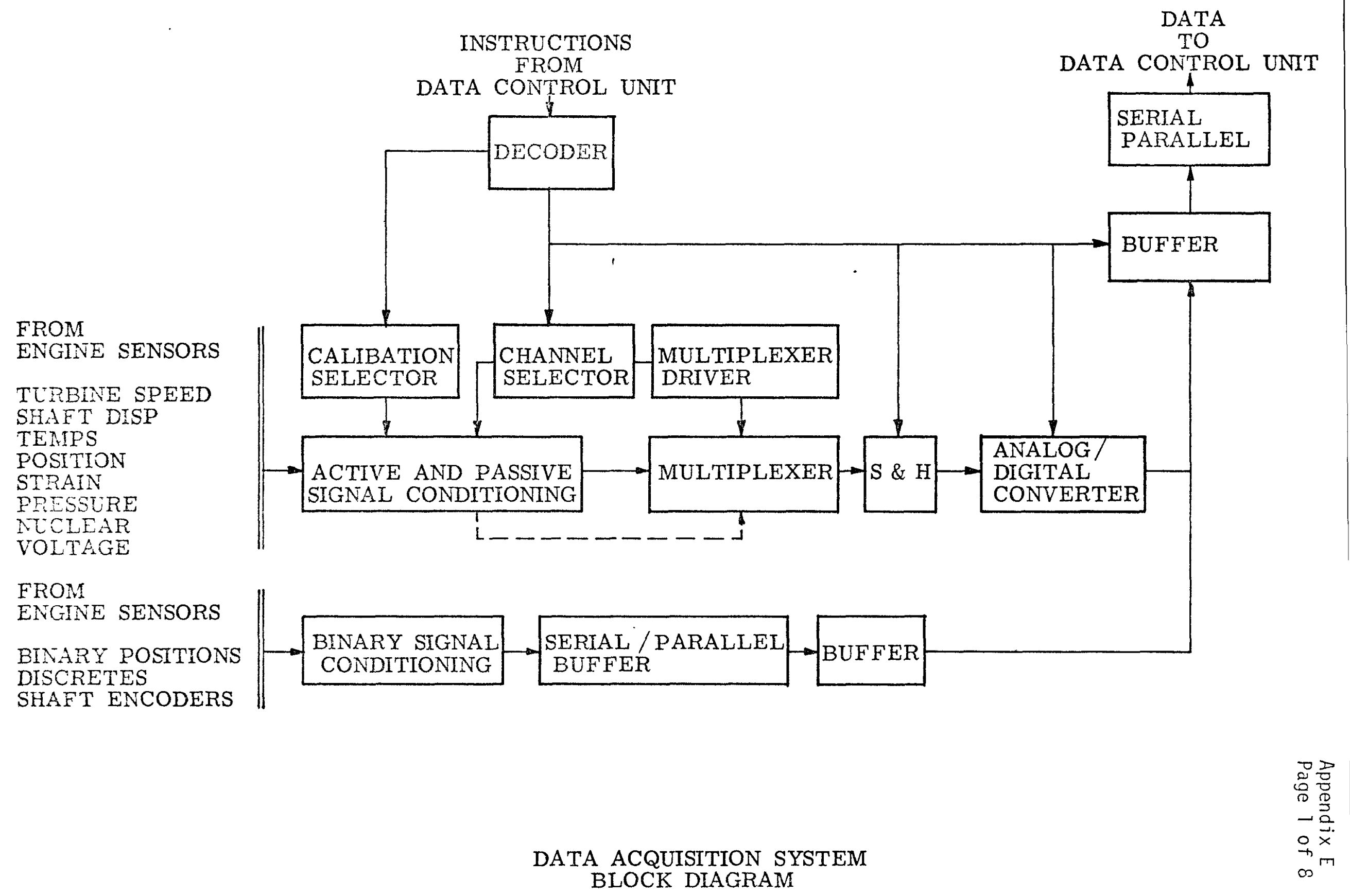




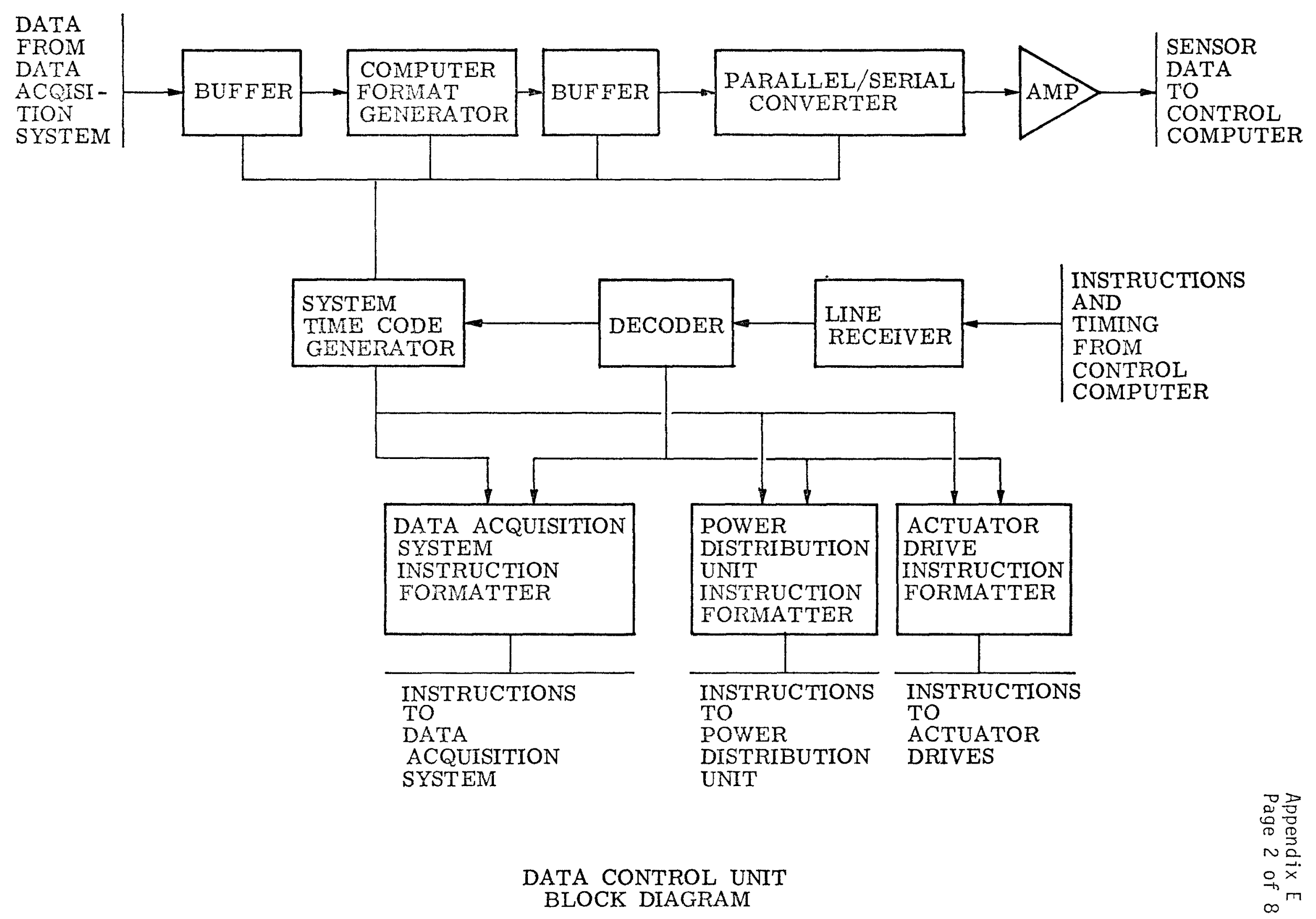




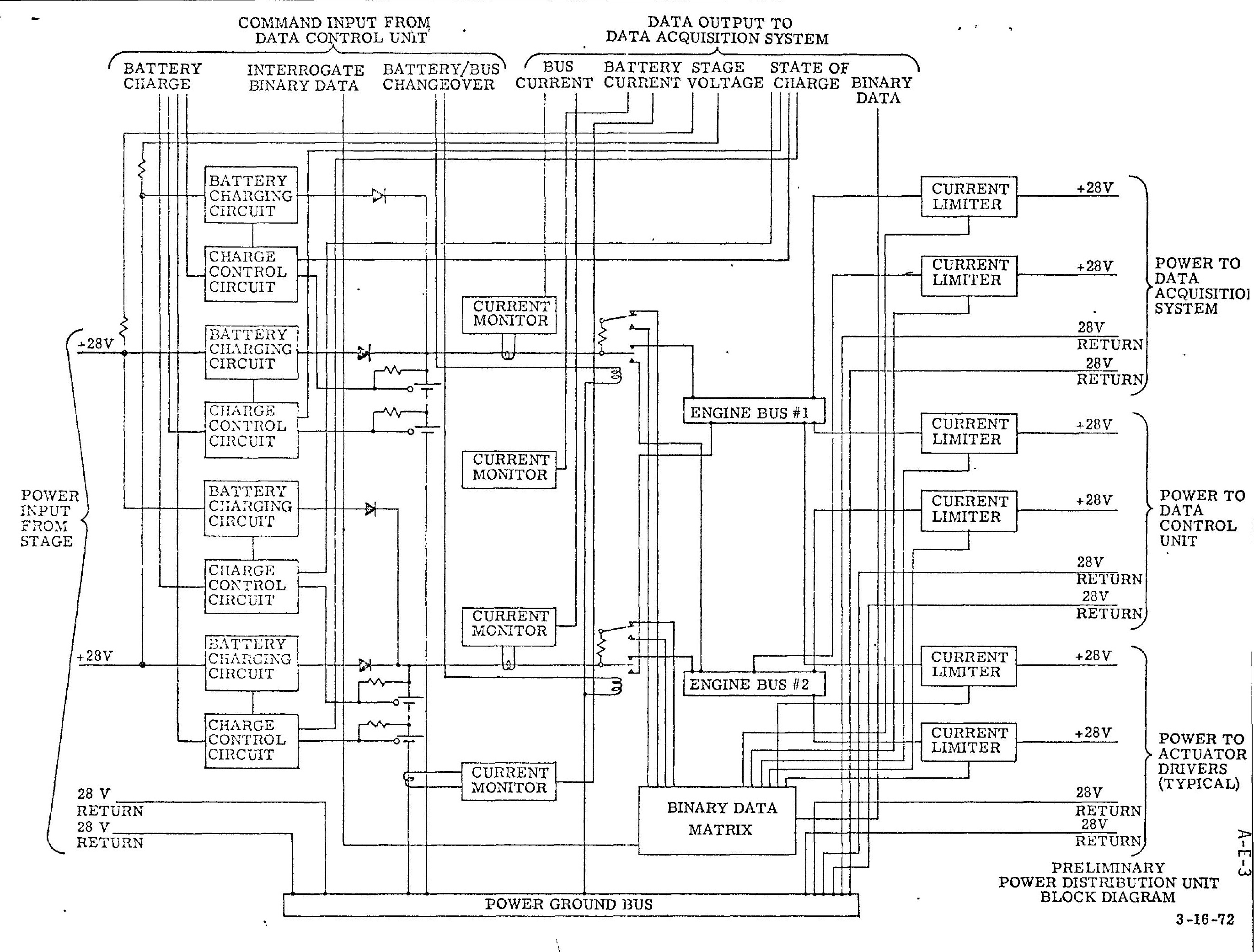




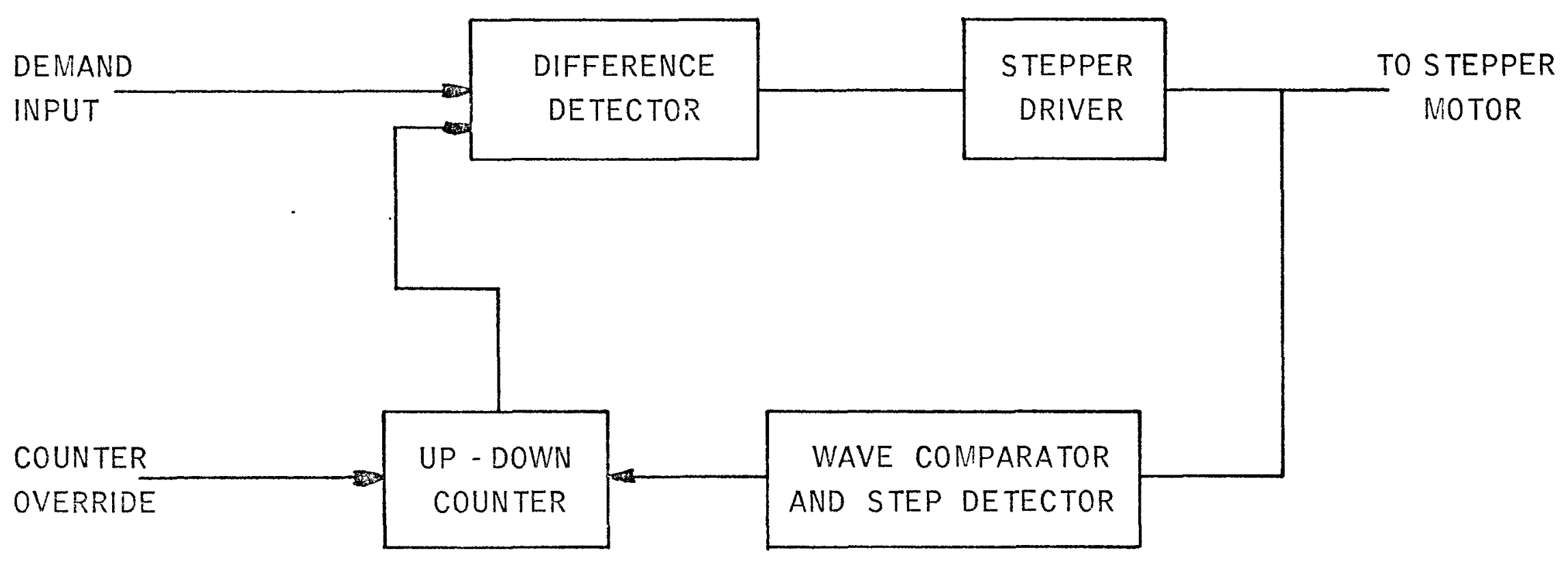

TYPICAL FOR 18 CDDA'S AND 2 SSCVA'S ACTUATOR DRIVER UNIT ${ }_{\infty}^{-m}$ CONTROL DRUN! \& SSCV 


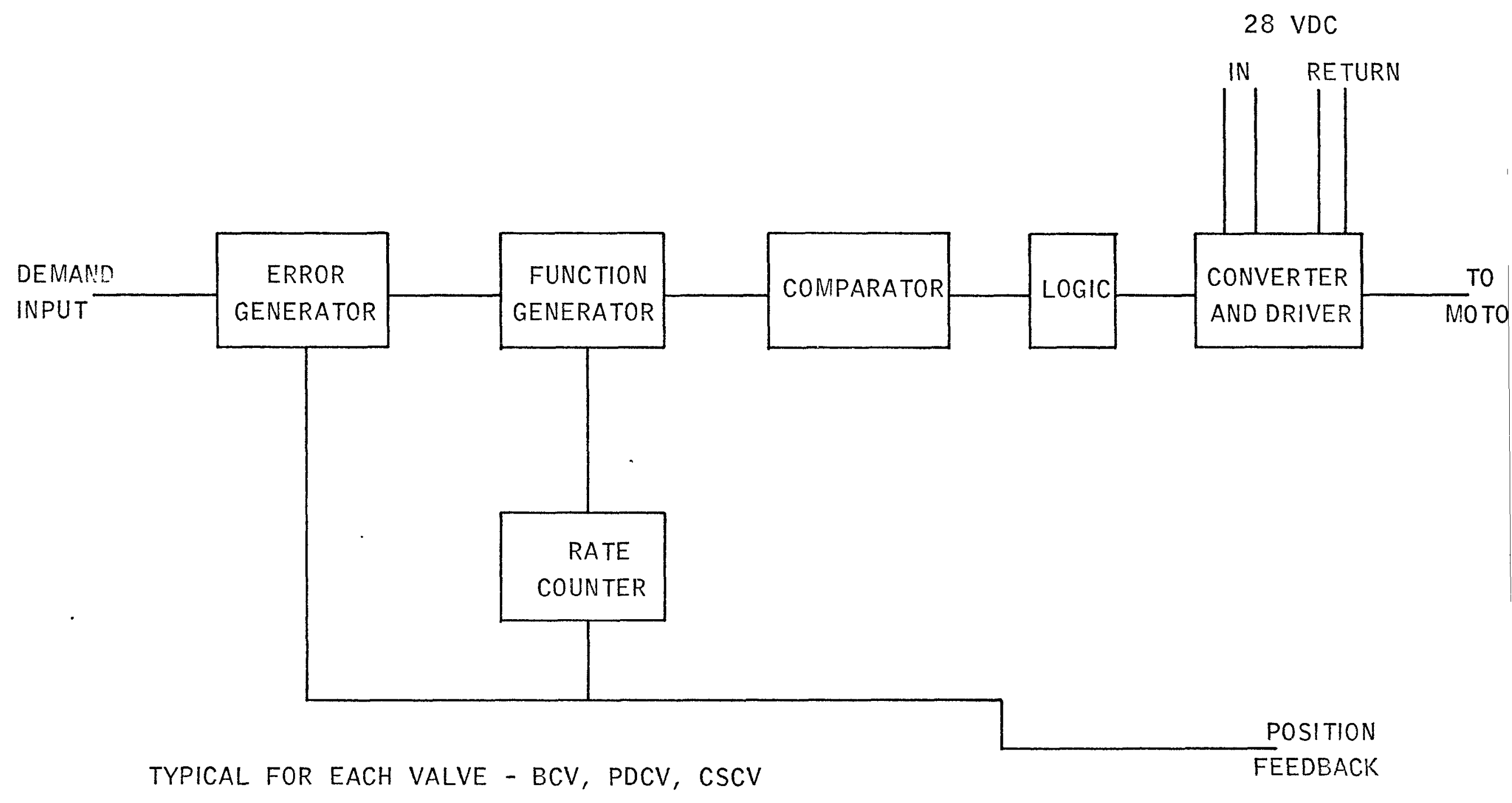

ACTUATOR DRIVER UNIT BCV, PDCV, CSCV PORTION

3-29- 72

SHEET 3 OF 6

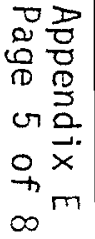




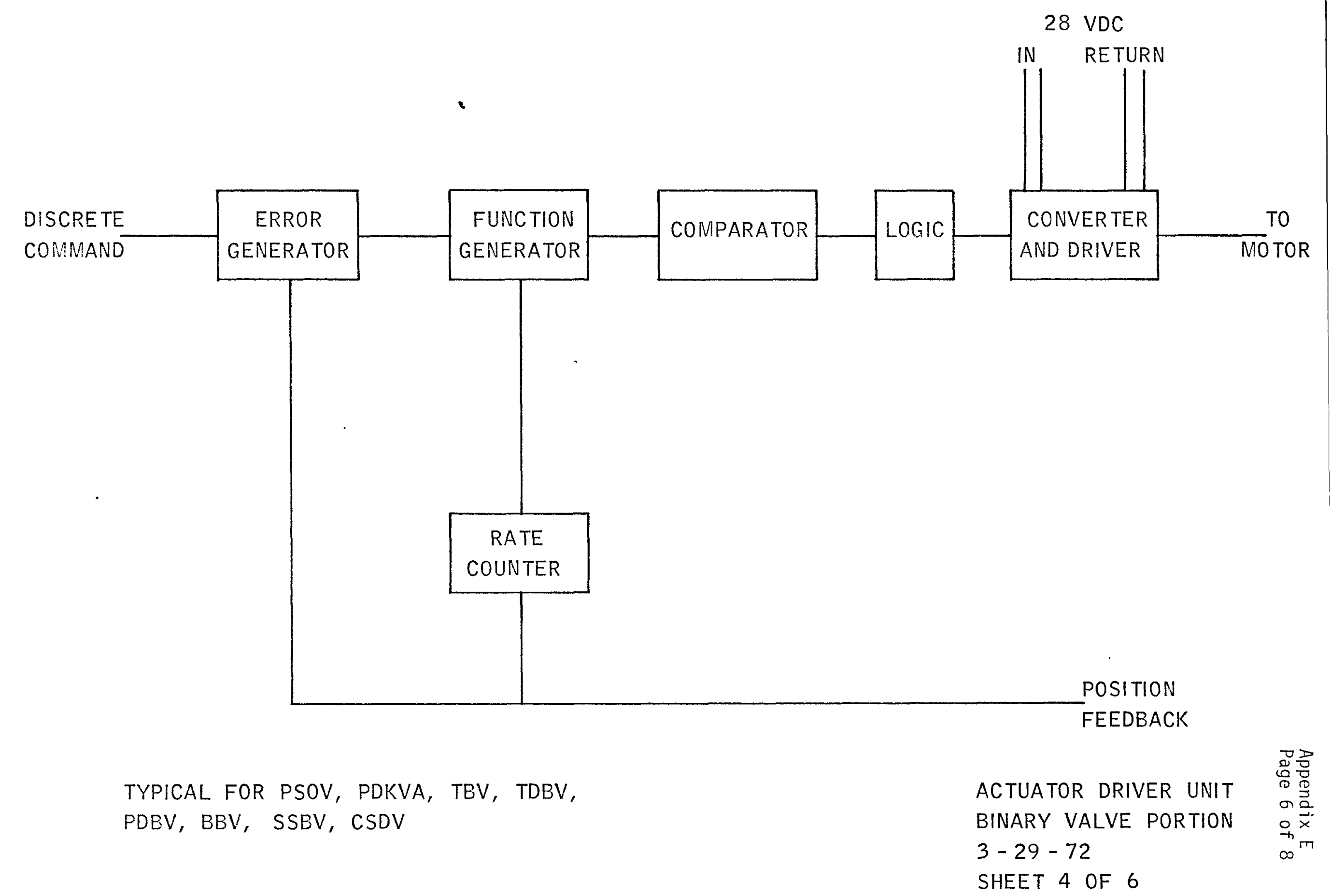




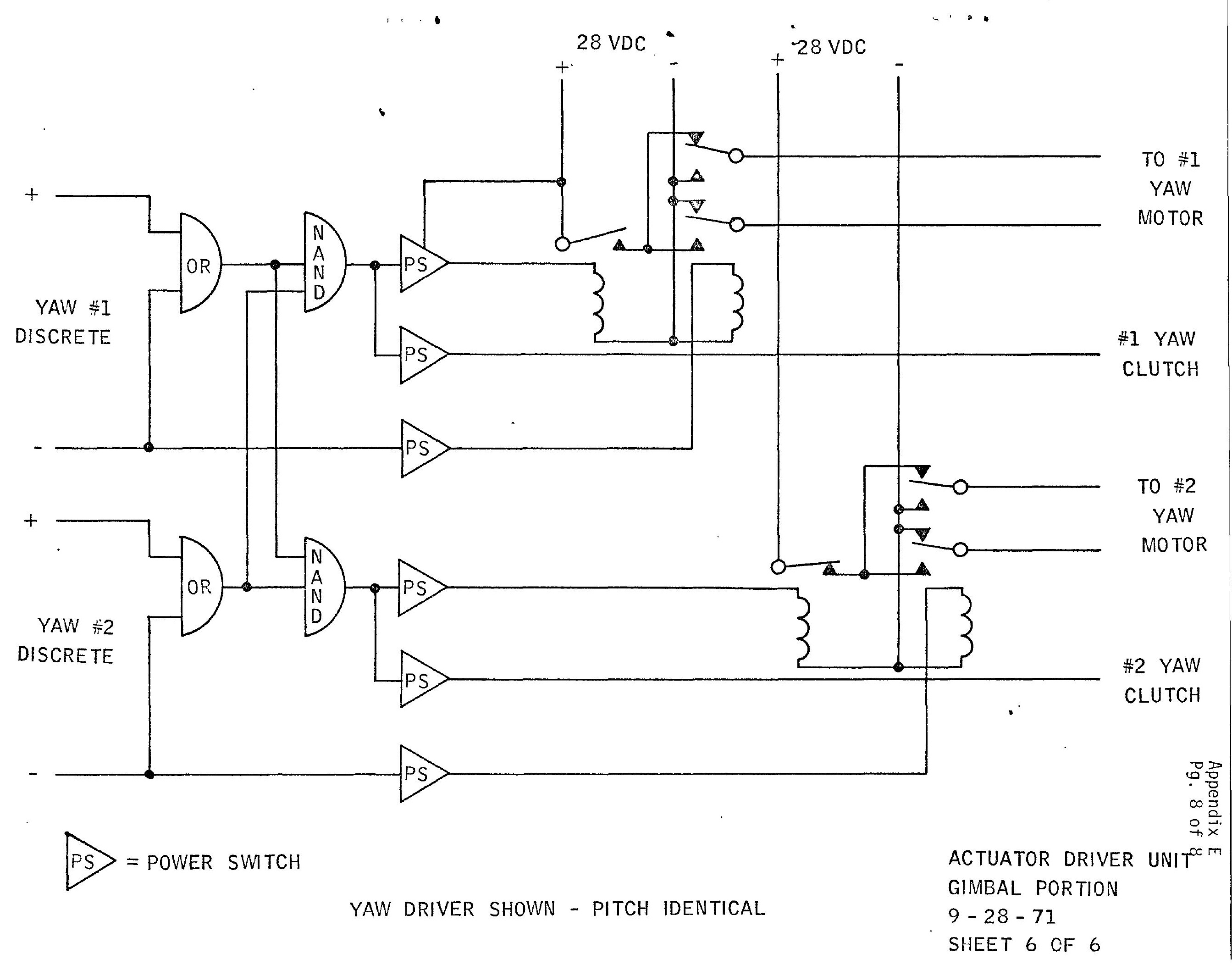

